Purdue University

Purdue e-Pubs

$1-1-2007$

\title{
Characterization and Optimization of the Thermal Performance of Miniature Piezoelectric Fans
}

Tolga Acikalin

S V. Garimella

Purdue University, sureshg@purdue.edu

Arvind Raman

James Petroski

Follow this and additional works at: http://docs.lib.purdue.edu/coolingpubs

Acikalin, Tolga; Garimella, S V.; Raman, Arvind; and Petroski, James, "Characterization and Optimization of the Thermal Performance of Miniature Piezoelectric Fans" (2007). CTRC Research Publications. Paper 52.

http://dx.doi.org/10.1016/j.ijheatfluidflow.2006.10.003

This document has been made available through Purdue e-Pubs, a service of the Purdue University Libraries. Please contact epubs@purdue.edu for additional information. 


\title{
Characterization and Optimization of the Thermal Performance of Miniature Piezoelectric Fans
}

\author{
Tolga Açıkalın*, Suresh V Garimella*, Arvind Raman* and James Petroski ${ }^{\text {II }}$ \\ *Purdue University, West Lafayette, Indiana, 47907-2088 USA \\ sureshg@ecn.purdue.edu

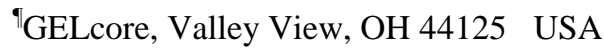 \\ Jim.Petroski@gelcore.com
}

\begin{abstract}
Piezoelectric fans have emerged as a viable cooling technology for the thermal management of electronic devices, owing to their low power consumption, minimal noise emission, and small and configurable dimensions. Piezoelectric fans are investigated for application in the cooling of low-power electronics. Different experimental configurations are considered, and the effect of varying the fan amplitude, the distance between the fan and the heat source, the fan length, its frequency offset from resonance, and the fan offset from the center of the heat source are studied to assess the cooling potential of the fans. A Design-Of-Experiments (DOE) analysis revealed the fan frequency offset from resonance and the fan amplitude as the critical parameters. Transfer functions are obtained from the DOE analysis for the implementation of these fans in electronics cooling. For the best case, an enhancement in convective heat transfer coefficient exceeding 375\% relative to natural convection was observed, resulting in a temperature drop at the heat source of more than $36.4^{\circ} \mathrm{C}$. A computational model for the flow field and heat transfer induced by the piezoelectric fan is also developed. Effects of the flow on convection heat transfer for different fan-to-heat source distances and boundary conditions are analyzed. Transition between distinct convection patterns is observed with changes in the parameters. The computational results are validated against experimental measurements, with good agreement.
\end{abstract}

Keywords: electronics cooling, piezoelectric fans, miniature fans, low-noise fans, heat transfer enhancement. 


\section{NOMENCLATURE}

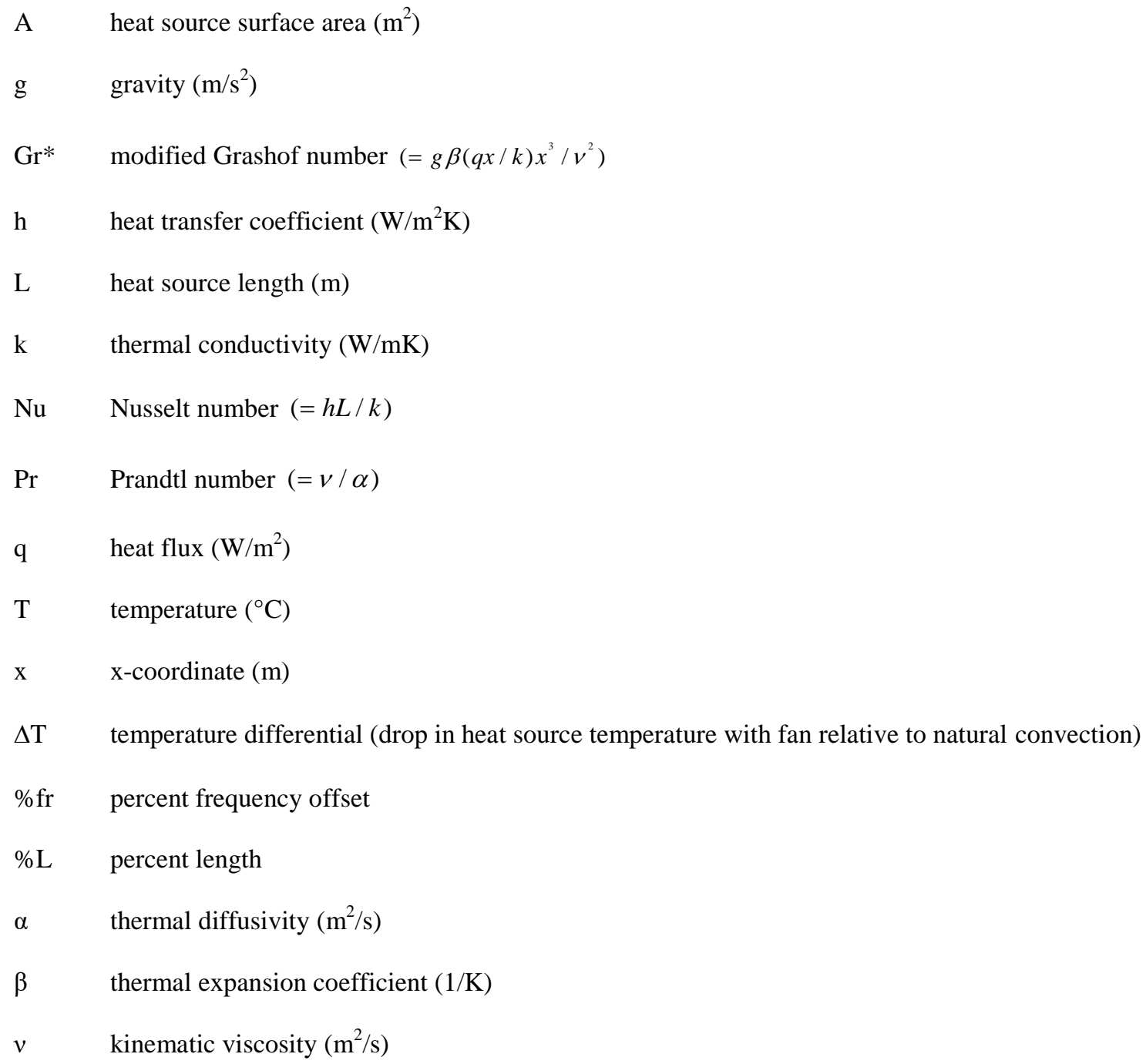




\section{INTRODUCTION}

Natural convection as a preferred means for thermal management in view of its simplicity of implementation is fast approaching its limits in the face of increasing heat dissipation from electronics in conjunction with consumer demand for smaller devices. Thermal management in laptops, cell phones, PDAs and LED packages are good examples of such situations. Although conventional methods for heat removal, such as incorporation of rotational fans are thermally viable, it is the minimized space, power, and noise requirements that point to piezoelectric fans as a preferred alternative for thermal management.

A piezoelectric fan is fabricated either by bonding a piezoelectric patch (or several patches) to a shim material, or by using the patch itself with no shim attached. Under an alternating voltage, which is applied to the electrodes of the piezoelectric patch, the patch expands and contracts alternately at the same frequency as the input signal. When attached to a shim material, this results in the shim flapping back and forth like a hand-held fan, but at a much higher frequency.

These fans are driven at resonance, meaning that the alternating voltage is applied at the frequency of a resonance mode of the piezoelectric fan. Driving the fan at resonance leads to a high value of the ratio of tip deflection to power consumption. To ensure silent operation, the fans are designed such that their operational mode of resonance (for the intensity of sound generated) is outside the range of frequencies audible to the human ear, i.e., less than $100 \mathrm{~Hz}$ for $25 \mathrm{~dB}$.

A number of studies on piezoelectric fans have been reported in the literature. Toda $(1979,1981)$ found that placing a piezoelectric fan on either side of a power transistor panel of a television receiver resulted in a $17^{\circ} \mathrm{C}$ decrease in temperature on the panel surface. Yoo et al. (2000) developed several types of piezoelectric fans using PZT, one of which resulted in a fan tip deflection of $3.55 \mathrm{~cm}$ and an air velocity of $3.1 \mathrm{~m} / \mathrm{s}$ measured $0.1 \mathrm{~cm}$ away from the fan tip. Schmidt (1994) employed the naphthalene sublimation technique in experiments to determine the local and average transfer coefficients on a vertical surface cooled by two piezoelectric fans resonating out of phase. Changing the distance between the fans and the surface, or the distance between fans, was found to noticeably change the transfer coefficients for the system.

Flows around the ends of oscillating flexible cantilevers were investigated by Ihara and Watanabe (1994). The discrete vortex method was used to numerically simulate the flow field, which was compared against flow visualizations. Açıkalın et al. (2003) developed a closed-form analytical solution to predict the two-dimensional 
streaming flow from an infinite vibrating beam. This solution was used to develop a computational flow model for a baffled piezoelectric fan vibrating at its first mode of resonance in an infinite medium. Experimentally mapped flow patterns were found to closely match those predicted by the model for the baffled fan. Loh et al. (2002) investigated the cooling effects of acoustic streaming from an ultrasonically vibrating beam. A $40^{\circ} \mathrm{C}$ temperature drop was observed on a heater which was initially at $98^{\circ} \mathrm{C}$. The beam vibrated at $28 \mathrm{kHz}$ with an amplitude of $25 \mu \mathrm{m}$.

Optimization of the structure of piezoelectric fans was investigated by Buermann et al. (2002) and Basak et al. (2005) who considered fans with two symmetrically placed piezoelectric patches and a piezoelectric patch on only one side, respectively. The studies focused on optimizing the electromechanical coupling factor (EMCF) of these fans.

The feasibility of using piezoelectric fans in small-scale electronics cooling applications was investigated by Açıkalın et al. (2004) and Wait et al. (2004). Flow visualization experiments were conducted to gain insight into the flow induced by these fans. The thermal performance of piezoelectric fans was investigated in two different experiments: a custom-built setup was used to quantify the cooling enhancement from a heat-dissipating component due to piezoelectric fans in an enclosure simulating a cellular phone, and a commercially available laptop computer was utilized to demonstrate the viability of using piezoelectric fans for localized cooling. In the first setup, the piezoelectric fans were found to offer enhancements in convective heat transfer coefficients of more than $100 \%$ relative to natural convection, while in the latter, a $6^{\circ} \mathrm{C}$ to $8^{\circ} \mathrm{C}$ temperature drop was observed in the electronic components within the laptop.

In the present study, the potential offered by piezoelectric fans for the thermal management of low-power electronics components is assessed experimentally as well as numerically. Three different experimental configurations are considered in order to map the effects of several system parameters on the heat transfer from a small heat source. The five parameters considered are the fan amplitude, the distance between the fan and the heat source, the fan length, its frequency offset from resonance, and the fan offset from the center of the heat source. A design of experiments (DOE) approach is used in the experiments. Transfer functions are obtained from the DOE analysis for the implementation of these fans in cooling applications.

A simplified computational model is also developed and the predictions compared against the experimental results. Effects of the flow generated on convection heat transfer for different fan-to-heat source distances and boundary conditions are analyzed. 
Piezoelectric fans are a very new technology and the basic design guidelines for implementing these fans into practical thermal solutions are not yet established. For example, for inclusion into a laptop, a large number of design variables must be optimized; these include fan operating frequency, distance from heat source, fan length amplitude, and off-resonance variability. Such trade-offs are further complicated by the fact that the physics controlled by each of these parameters is coupled: for instance, higher-frequency operation may increase fluid flows only if the fan-surface distance is larger than a certain value. Clearly the coupled, three-dimensional, unsteady nature of the mechanical, temperature and flow fields involved in such a device poses significant challenges to the rational design and integration of piezoelectric fans into portable electronic devices. In this regard, the work presented in this article is expected to provide initial design guidelines for the practical design and implementation of piezoelectric fans into commercial electronic devices.

\section{EXPERIMENTAL SETUP AND PROCEDURES}

A typical electronics package is mimicked in the experiments as a constant heat flux source. The heat source was constructed by attaching a kapton thin-film heater to the back of an aluminum plate of dimensions $3.81 \mathrm{~cm} \times$ $3.81 \mathrm{~cm} \times 0.2 \mathrm{~cm}$. This assembly was embedded in styrofoam insulation, leaving the heat source flush with the insulation surface (Figure 1), to minimize heat losses from the back and sides of the heat source. The exposed surface of the aluminum plate was painted with Krylon \#1602, which has a known emissivity of $0.95^{1}$.

Fourteen 36-gage type-T thermocouples were used to monitor temperatures in the experiments. Of these, five were mounted on the exposed surface of the heat source (as shown in Figure 1, to calculate the average surface temperature), one was sandwiched between the heater and the insulation, and another was attached to the backside of the insulation to provide an estimate of the heat loss. One thermocouple was used to monitor the ambient air temperature in the enclosure remote from the heat source. The remaining six thermocouples were suspended in air, four near the heat source, and two near the fan, as shown in Figure 1. For the finned heat source, three additional thermocouples were inserted into the fin.

\footnotetext{
${ }^{1}$ NASA Jet Propulsion Laboratory Web Site, URL: http://masterweb.jpl.nasa.gov/reference/paints.htm
} 
A large transparent box $(36.6 \mathrm{~cm} \times 36.6 \mathrm{~cm} \times 36.6 \mathrm{~cm})$ was placed around the heat source and fan setup as shown in Figure 2 to isolate the assembly from extraneous convection currents in the room. Both the test setup and the piezoelectric fan being studied were mounted on an optical rail to allow for precise relative positioning.

Three different configurations of the piezofan and heat source were studied as shown in Figure 3 . In the first two configurations, the position of the fan relative to the heat source was changed, while in the third, a fin was included on the heat source, with the fan position being held the same as in the first case. The fin in the third configuration was $0.15 \mathrm{~cm}$ thick and $0.64 \mathrm{~cm}$ high, and extended the entire length of the heat source.

For each of the configurations, the heat transfer coefficient was first measured under natural convection conditions, to serve as a baseline. The enhancement of heat transfer upon turning on the piezoelectric fan was then measured and compared to the baseline free convection results.

Five different parameters were varied in the experiments. The frequency offset from the resonance frequency was varied by $\pm 5 \%$. Three values of input voltage were studied, resulting in three different ratios of tip deflection to fan length under resonance conditions (amplitude ratios) ranging from 10 to $25 \%$. It may be noted that because of this definition of amplitude ratio, the fan amplitude under a frequency offset is lower than its resonance value. The normal distance between the heat source and fan tip at rest (distance to fan tip) as well as the distance along the heat source between fan tip and heat source center were two other parameters, as illustrated in Figure 3 . Finally, two piezoelectric fans of different lengths were used to investigate the effect of fan length. This, of course, results in a difference in their resonance frequencies $(62 \mathrm{~Hz}$ and $103 \mathrm{~Hz}$, respectively for the $7.62 \mathrm{~cm}$ and $6.86 \mathrm{~cm}$ fans).

The uncertainity in the measurement of geometric parameters (fan length, fan offset and distance to fan tip) is $0.1 \mathrm{~mm}$. The amplitude of the fan is measured with the help of calipers and the uncertainity in this measurement is estimated to be $0.5 \mathrm{~mm}$. The error in the temperature measurement is estimated to be within $\pm 0.75^{\circ} \mathrm{C}$. The total power input to the heat source is calculated as a product of the voltage drop across the heater and the current passing through it, and has an estimated uncertainity of $\pm 0.02 \mathrm{~W}$; this includes contributions from the multimeter as well as electrical losses in the lead wires.

The large number of parameters affecting the heat transfer in this experiment suggested the use of a design of experiments (DOE) approach (Schmidt and Launsby, 2003). This approach offers key advantages over typical experimentation methods when several variables are under investigation. The conventional approach is to vary one experimental factor at a time (OFAT) while holding other parameters fixed. This usually does not readily lead to an 
optimum solution, nor does it allow parameter interactions to be explored. As a result, the goal of optimizing an output, such as heat transfer, may not be realized by the OFAT approach. The DOE process, in contrast, allows for optimal values of the parameters to be predicted, even if actual experiments are not conducted with these values.

Also, a properly constructed DOE can map out transfer functions and response surfaces with fewer experimental runs than by any other methodology. The resolution between the variables is determined by the type of DOE setup that is chosen; the higher the resolution of the DOE, the less is the "confounding" or indistinguishability between combinations of variables. Once a resolution level is chosen, the DOE matrix provides the fewest number of experiments to create the transfer function. Thus a DOE not only provides the best information about a system, but also does so most economically.

\section{EXPERIMENTAL RESULTS AND DISCUSSION}

Experimental results for Configuration A are first discussed in detail, with results for the other two configurations summarized next.

\section{Configuration A}

Results for a sample experiment in this configuration are shown in Figure 4. Measured temperatures for a test with a $7.62 \mathrm{~cm}$ long fan operating at resonance with a $25 \%$ amplitude ratio (i.e., amplitude-to-fan length ratio) are shown. The distance to the fan tip is $0.64 \mathrm{~cm}$ and there is no fan offset in this test. In this configuration the total power input to the heat source was $1.6 \mathrm{~W}$, at which the average surface temperature of the heat source under natural convection conditions was $70^{\circ} \mathrm{C}$. The power input was corrected for the heat loss through the insulation (which was approximately $0.09 \mathrm{~W}$ in natural convection and $0.03 \mathrm{~W}$ with the fan operational) in the calculation of heat transfer coefficients. Readings from the fixed thermocouples (see Figure 1) are shown in Figure 4(a), while those from thermocouples suspended in air are shown in Figure 4(b). At $t=0$ the heater is turned on and at $t \approx 1500 \mathrm{~s}$, the system reaches a steady state in natural convection. The piezoelectric fan is turned on at $\mathrm{t}=2000 \mathrm{~s}$ and a new steady state is reached after approximately $500 \mathrm{~s}$. The fan is seen to cause a reduction in the average heat source temperature from approximately $70.6^{\circ} \mathrm{C}$ in natural convection to $37.4^{\circ} \mathrm{C}$ with the piezofan. The increase in heat transfer coefficient due to the fan causes an increase in the measured air temperatures around the heat source. The 
heat transfer coefficient in these experiments is defined based on the difference between the average heat source surface temperature and the ambient enclosure temperature.

Similar measurements were obtained for a DOE matrix consisting of a total of 30 experiments for Configuration A. The lower and upper specification limits (LSL and USL) for the five parameters considered are shown in Table 1. The steady-state heat transfer coefficients for three individual experiments from the DOE matrix in Configuration A are shown in Figure 5. The heat transfer coefficients shown in Figure 5 are effective values, including both convective and radiative effects, and computed as:

$$
\begin{aligned}
& \mathrm{h}=\frac{\left(\mathrm{q}_{\text {in }}-\mathrm{q}_{\text {loss }}\right)}{\mathrm{A}\left(\mathrm{T}_{\mathrm{s}}-\mathrm{T}_{\infty}\right)} \\
& \mathrm{q}_{\text {loss }}=\frac{\mathrm{k}_{\text {ins }} \mathrm{A}_{\text {ins }}\left(\mathrm{T}_{\mathrm{h}}-\mathrm{T}_{\text {ins }}\right)}{\mathrm{L}_{\text {ins }}}
\end{aligned}
$$

In Eq. (1) $q_{\text {in }}$ is the heat input to the heat source, $q_{\text {loss }}$ the loss from the heat source, $A$ is the heat source area, $A_{\text {ins }}$ is the surface area of the insulation block that is in contact with the heat source (four sides of the plate as well as its backside), $\mathrm{k}_{\text {ins }}$ is the thermal conductivity of the insulation block in which the heat source is embedded, and $\mathrm{L}_{\text {ins }}$ is the thickness of the insulation block. Furthermore $T_{s}, T_{h}, T_{\text {ins }}$, and $T_{\infty}$ denote average heat source surface temperature, temperature in between the heat source and insulation block, backside insulation block surface temperature, and ambient air temperature, respectively. Thermal losses from the heat source is assumed to be onedimensional and varied depending on the final average heat source temperature reached in individual experiments. Thermal loss from the heat source was $0.03 \mathrm{~W}$ for the case with best piezofan cooling, and was $0.09 \mathrm{~W}$ under natural convection.

Also shown in Figure 5 is the effective heat transfer coefficient, computed in the same manner. When the piezofan fan is turned off, natural convection and radiation are the two modes of heat transfer from the heat source, and an effective heat transfer coefficient of $21.62 \mathrm{~W} / \mathrm{m}^{2} \mathrm{~K}$ is obtained under these conditions.

The average heat transfer coefficients for the 30 experiments in Configuration A varied from approximately 23 to $80 \mathrm{~W} / \mathrm{m}^{2} \mathrm{~K}$. Even in the worst case (Figure 5), an approximately $7 \%$ enhancement over natural convection was seen. For the best case, an enhancement of greater than $275 \%$ over natural convection was achieved. Results from the DOE analysis are presented in the following as a number of surface plots, showing the effects of changes in the governing parameters on the temperature drop achieved on the heat source with the fan relative to the temperatures 
under natural convection conditions. The frequency offset is revealed to be the most important of the five parameters considered. As the fan is operated farther away from resonance, its amplitude suffers greatly. Since a lower amplitude causes less airflow, the cooling capacity of the fan decreases correspondingly. Figure 6a shows the influence of frequency offset and fan distance on the temperature differential achieved at the heat source. A frequency offset of $5 \%(3.1 \mathrm{~Hz})$ is seen to decrease the cooling capacity of the fan by $10^{\circ} \mathrm{C}$ (under optimal values for the remaining four factors). Moving the fan further away from the heat source resulted in an expected drop in performance: the extreme positions of the fan considered caused a difference of approximately $10^{\circ} \mathrm{C}$ in the temperature of the heat source. It may be noted that the optimum value of fan-to-tip distance resides outside the DOE tests, and heat transfer from the surface would increase as the distance is reduced below $0.64 \mathrm{~cm}$; this is consistent with the observations in Schmidt (1994). As for the other parameters (see Figure 6b), doubling the fan amplitude (from $12.5 \%$ to $25 \%$ of fan length) reduces the temperature of the heat source by $16.4^{\circ} \mathrm{C}$. Although displacing the fan to one side (increasing the offset) decreases the cooling achieved, this effect is much smaller than the other three factors discussed.

In the context of the experiments performed here, the shorter fan always performs better than the longer fan. The two fans of different length have quite different resonance frequencies $(62 \mathrm{~Hz}$ and $103 \mathrm{~Hz}$, respectively, for the $7.62 \mathrm{~cm}$ and $6.86 \mathrm{~cm}$ fans). This difference in resonance frequencies, in conjunction with the amplitudes of vibration being quite similar in the two cases, results in higher fluid velocities in the case of the shorter fan. The temperature differential is found to be $10 \%$ to $20 \%$ greater when the shorter fan is used; the temperature differential is affected more strongly at the lower fan amplitudes.

The transfer function obtained from the DOE analysis for the temperature drop on the heat source in Configuration $\mathrm{A}$ is given below:

$$
\begin{aligned}
\Delta \mathrm{T} & =-3.26 \cdot \mathrm{R}_{\mathrm{A}}+6.60 \cdot \mathrm{R}_{\mathrm{B}}-0.87 \cdot \mathrm{R}_{\mathrm{C}}-0.21 \cdot \mathrm{R}_{\mathrm{D}} \\
& -2.77 \cdot \mathrm{R}_{\mathrm{E}}-0.91 \cdot \mathrm{R}_{\mathrm{B}} \cdot \mathrm{R}_{\mathrm{E}}-7.91 \cdot \mathrm{R}_{\mathrm{C}}{ }^{2} \\
& -0.44 \cdot \mathrm{R}_{\mathrm{C}} \cdot \mathrm{R}_{\mathrm{D}}+0.48 \cdot \mathrm{R}_{\mathrm{D}} \cdot \mathrm{R}_{\mathrm{E}}+26.58
\end{aligned}
$$

in which $\mathrm{R}_{\mathrm{i}}$ are the standardized parameters in the order shown in Table $1\left(\mathrm{R}_{\mathrm{A}}\right.$ represents fan length, etc.). In this context, "standardized" means -1 for the lower specification limit (LSL), 1 for the high limit (USL), and 0 for the mean value. This model predicts the experimental observations with a correlation coefficient $\mathrm{R}^{2}$ of 0.98 , and maximum and average deviations in $\Delta \mathrm{T}$ of $2.04^{\circ} \mathrm{C}$ and $0.71^{\circ} \mathrm{C}$, respectively. 
In the construction of the model presented in Eq. (2), the five parameters shown in Table 1 as well as all of their two-way interactions, including the quadratic interaction terms, are considered first. Using the commercial software, DOE KISS ${ }^{2}$, a multiple-regression least-squares fit is used to determine the coefficients in the full model. The probability that a term does not belong in the regression model is also calculated for every term. Starting with the lowest probable interaction, terms in the general model are eliminated one by one, until every term remaining in the model had a confidence value greater than $90 \%$ (or P $<0.1$ ). After the elimination of each term from the model a new regression analysis is performed. The cut-off value of $90 \%$ for the confidence level is suggested in standard DOE texts (such as Schmidt and Launsby, 2003).

In view of the predictions from the DOE results, an additional experiment was conducted at the "best predicted" point (with the five parameter values set at the optimal values which maximize $\Delta \mathrm{T}$ in Eq. (4)) and an enhancement of more than $375 \%$ over natural convection was achieved for this orientation, matching the transfer function model. This best performance is obtained at a fan length of $6.86 \mathrm{~cm}$, amplitude ratio of $25 \%$, fan-to-heat source distance of $0.64 \mathrm{~cm}$ and without any frequency or fan offset. Under these conditions a temperature differential of $36.4^{\circ} \mathrm{C}$ and an average heat transfer coefficient of $100.8 \mathrm{~W} / \mathrm{m}^{2} \mathrm{~K}$ were achieved.

\section{Configuration B}

In Configuration B, the piezofan was placed parallel to the heat source (Figure 3). As shown in Table 1, the only parameters that were given different LSL and USL values when compared to Configuration A, were the fan offset and distance between the heat source and fan. Also, the distance between the heat source and fan tip in this configuration is referenced from the deflected fan position when the tip is closest to the heat source (Distance A in Figure 3).

The results of the DOE analysis for this configuration are shown in Figure 7. It is again clear from these results that the frequency offset is the most important determinant of heat transfer. As the fan is operated farther offresonance, its amplitude decreases as does its cooling capability. Figure 7 shows a decrease in cooling capacity of more than $15^{\circ} \mathrm{C}$ for a frequency offset of $5 \%$. As in Configuration $\mathrm{A}$, moving the fan further away from the heat source results in a performance drop. In contrast to Configuration A, however, the fan offset plays a more

\footnotetext{
${ }^{2}$ DOE KISS, Air Academy Associates, Colorado Springs, CO.
} 
significant role in Configuration B, and causes a drop in temperature differential on the heat source of approximately $12^{\circ} \mathrm{C}$, when compared to the original fan position.

The following transfer function is obtained from the DOE analysis, conducted as explained previously, for the temperature drop on the heat source in Configuration B:

$$
\begin{aligned}
\Delta \mathrm{T} & =-2.07 \cdot \mathrm{R}_{\mathrm{A}}+4.87 \cdot \mathrm{R}_{\mathrm{B}}-1.06 \cdot \mathrm{R}_{\mathrm{C}} \\
& -2.66 \cdot \mathrm{R}_{\mathrm{D}}-0.88 \cdot \mathrm{R}_{\mathrm{E}}-1.69 \cdot \mathrm{R}_{\mathrm{B}} \cdot \mathrm{R}_{\mathrm{D}} \\
& -8.25 \cdot \mathrm{R}_{\mathrm{C}}{ }^{2}-0.62 \cdot \mathrm{R}_{\mathrm{C}} \cdot \mathrm{R}_{\mathrm{D}}+23.79
\end{aligned}
$$

This model predicts the experimental observations with a correlation coefficient $R^{2}$ of 0.96 , and maximum and average deviations in $\Delta \mathrm{T}$ of $3.86^{\circ} \mathrm{C}$ and $1.4^{\circ} \mathrm{C}$, respectively.

\section{Configuration C}

This configuration is identical to Configuration A, with the main difference being that instead of a flat heat source, an aluminum fin is present on the surface as explained earlier (Figure 3). Because of the increased surface area, the power input is increased from the value used in Configurations A and B to achieve the same surface temperature on the heat source as before. A power input of $1.8 \mathrm{~W}$ is required in this case to maintain an average heat source surface temperature of $70^{\circ} \mathrm{C}$ under natural convection conditions.

Table 1 shows the LSL and USL values for the parameters studied in this configuration. One common parameter between all three configurations is the fan to heat source distance, which is $0.64 \mathrm{~cm}$. It is noted that in Configuration C, this distance is measured not from the plane of the heat source but instead from the tip of the fin, making the total distance from the heat source plane $1.27 \mathrm{~cm}$.

As seen from Figure 8, Configuration $\mathrm{C}$ has similar trends as Configuration $\mathrm{A}$. In Configuration $\mathrm{C}$, the temperature differentials are slightly lower than those in Configuration A; however, Configuration C has 13\% greater heat dissipation. Due to the presence of the fin, the fan offset parameter was not investigated and the fan was

always centered with respect to the finned heat source. Thus the transfer function derived does not contain any fan offset terms $\left(\mathrm{R}_{\mathrm{D}}\right)$ :

$$
\begin{aligned}
\Delta \mathrm{T} & =-3.33 \cdot \mathrm{R}_{\mathrm{A}}+7.33 \cdot \mathrm{R}_{\mathrm{B}}-0.95 \cdot \mathrm{R}_{\mathrm{C}} \\
& -2.11 \cdot \mathrm{R}_{\mathrm{E}}-0.99 \cdot \mathrm{R}_{\mathrm{A}} \cdot \mathrm{R}_{\mathrm{B}}-0.67 \cdot \mathrm{R}_{\mathrm{A}} \cdot \mathrm{R}_{\mathrm{C}} \\
& -0.94 \cdot \mathrm{R}_{\mathrm{B}} \cdot \mathrm{R}_{\mathrm{E}}+7.91 \cdot \mathrm{R}_{\mathrm{C}}{ }^{2}+25.89
\end{aligned}
$$


This model predicts the experimental observations with a correlation coefficient $R^{2}$ of 0.98 , and maximum and average deviations in $\Delta \mathrm{T}$ of $2.3^{\circ} \mathrm{C}$ and $0.98^{\circ} \mathrm{C}$, respectively.

\section{Comparison of Configurations}

In Figure 9, DOE results from Figures 6, 7 and 8 are plotted together to facilitate comparison. From this figure it is seen that the temperature differential is highest for Configuration A and lowest for Configuration B. In all three configurations, the frequency dependence of the temperature differential is similar. However the effect of fan distance on the temperature differentials is quite different: Configurations $\mathrm{A}$ and $\mathrm{C}$ are more strongly dependent (have a higher slope) on the fan distance than Configuration B.

Although Configuration $\mathrm{C}$ has a lower temperature differential than Configuration $\mathrm{A}$, it has higher average heat transfer coefficients. This is illustrated for three comparable experiments for the two configurations in Figure 10. The use of a heat transfer coefficient accounts for the difference in heat inputs in the two orientations. Plotted in this manner, it is clear that inclusion of the fin enhanced the heat transfer significantly. This increase in heat transfer coefficient is in spite of an increase in the heat source to fan distance.

In Figure 11, temperature differentials achieved on the heat source in Configurations A and B are compared for different fan amplitude and fan offset. Since there is no fan offset in Configuration C, it is not included in this figure. Also, the fan offset in Configuration $\mathrm{A}$ is in only one (i.e., positive) direction, since this parameter is symmetric with respect to the centerline of the heat source in this orientation. The figure shows that the dependence of the temperature differential in Configuration A on fan amplitude is stronger than in Configuration B. On the other hand, the dependence on the fan offset is much greater in Configuration B.

\section{COMPUTATIONAL MODEL}

A computational model is developed to predict the flow field generated by the piezoelectric fan as well as the heat transfer achieved at the heat source. In the model, the actual three-dimensional geometry of the experimental setup is simplified to a two-dimensional one as shown in Figure 12. The two-dimensional computational model considers the horizontal mid-plane through the experimental setup of Configuration A, as illustrated in the figure. Since gravity is normal to this two-dimensional plane, buoyancy effects cannot be included in the model. 
Furthermore, the size of the enclosure in the computations is somewhat smaller than the one used in the experiments $(30 \mathrm{~cm} \times 30 \mathrm{~cm}$ instead of $36.6 \mathrm{~cm} \times 36.6 \mathrm{~cm}$ in the experiment), thus reducing the required size of the domain by more than $40 \%$. The four sides of the enclosure shown in Figure 12 are set as isothermal walls in the numerical model. The clamp of the piezoelectric fan is neglected in the computational model, and instead replaced by a simple adiabatic baffle (wall) as shown in the figure. The piezoelectric fan boundary condition is modeled as a moving adiabatic wall whose location in time is set by a user defined function in FLUENT. The details of the piezofan boundary condition are discussed in the next section. Both the baffle and piezoelectric fan are modeled as infinitesimally thin walls with no thermal conduction allowed through them.

The enclosure includes a fixed heat source insulated on all lateral sides and on the back, as described in the experiments. Corresponding to the heat dissipation from the heat source in Configuration A, a uniform heat flux of

$1100 \mathrm{~W} / \mathrm{m}^{2}$ is applied to the exposed uninsulated wall of the heat source in the numerical model (as shown in Figure

12.) The other three walls of the heat source are considered to be adiabatic.

Three cases from the experiments in Configuration A were considered for the computations. The parameters varied in these cases were the fan to heat source distance and the fan amplitude to length ratio at resonance (Table 2). The other three parameters investigated in the experiments - the fan length, frequency offset from resonance, and the fan offset from the center of the heat source - were not explored in the computations. The initial fluid temperature and the temperature of the enclosure walls were set to $24^{\circ} \mathrm{C}$ in Cases 1 and 2 , and $23^{\circ} \mathrm{C}$ in Case 3 matching the ambient temperatures recorded in the three corresponding experiments.

\section{Simulation of the Piezofan}

A slender beam model for the displacement of the piezoelectric fan was presented in Basak et al. (2005). The first mode shape (resonance frequency of $60 \mathrm{~Hz}$ ) of a commercial piezoelectric fan with the dimensions and material properties shown in Table 3 was given in Basak et al. (2005) as:

$$
\begin{aligned}
& G_{1}(x)=a_{1} e^{-b_{1} x}+a_{2} e^{b_{1} x}+a_{3} \cos \left(b_{1} x\right)+a_{4} \sin \left(b_{1} x\right) \\
& \text { for } \quad 0 \leq x \leq L_{1} \\
& G_{2}(x)=a_{5} \cos \left(b_{2} x\right)+a_{6} \cosh \left(b_{2} x\right)+a_{7} \sin \left(b_{2} x\right)+a_{8} \sinh \left(b_{2} x\right) \\
& \text { for } \quad L_{1} \leq x \leq L_{2}
\end{aligned}
$$

where $L_{l}$ is the patch length and $L_{2}$ is the total fan length. The constants in the above beam deflection equation are: 


$$
\begin{array}{cccc} 
& & a_{1}=0.072861 & a_{5}=-0.4742 \\
L_{1}=0.029 & b_{1}=32.8682 & a_{2}=0.015744 & a_{6}=1.7902 \\
L_{2}=0.065 & b_{2}=49.4542 & a_{3}=-0.0886 & a_{7}=-0.3694 \\
& & a_{4}=0.05711 & a_{8}=-1.75577
\end{array}
$$

This mode shape is scaled to achieve the desired amplitude. These equations are used to define beam location during vibration by employing a user-defined function in the commercial software package FLUENT ${ }^{3}$.

The resonance frequency is rounded to $62.5 \mathrm{~Hz}$ in the computational model to achieve numerical stability, since it corresponds to a period of $0.016 \mathrm{~s}$. The error involved in using the beam-location equations above derived for a $60 \mathrm{~Hz}$ resonance frequency for a fan of slightly higher resonance frequency $(62.5 \mathrm{~Hz}$ in the numerical model, and 62 $\mathrm{Hz}$ in the experiments) is assumed to be small. The fluid velocities along the piezofan beam are determined from a time derivative of the above displacement equations in the software program.

\section{Mesh Generation}

The complexity of the moving wall boundary condition considered required a mesh in the incompressible fluid that would accommodate a shifting of the boundary. The significant displacement of the nodes at the large amplitudes considered rendered this task challenging. In order to reduce the number of deforming cells required, the fluid domain (shown in Figure 13) was divided into two regions by the introduction of a fluid interface, as indicated by the dash dot line in the detailed inset on the right in the figure. Region 'a' of the fluid domain includes the deforming beam. Fluid cells surrounding the beam are prescribed to adjust their size during beam movement. This mesh motion is the primary enabler of the large beam-deflection boundary condition. However, during beam and fluid cell movement, the fluid cells can be greatly skewed from the equilateral triangular conditions shown, resulting in damaged fluid cells for individual control volume analysis. The division of the domain by an interface as described reduces the number of deforming cells, since the cells only on one side of the interface are flagged as deforming. Along the interface, the fluid cells in region 'a' are allowed to translate in the vertical direction as the beam deflects to its extreme positions. A single cell spacing was included from the tip of the beam to the fluid interface. The presence of more than one cell in this location was found to introduce fluid cells that fail due to stretching; inclusion of no cell at this location, on the other hand, caused excessive stretching of the cells 
immediately adjacent to the deflecting beam. Thus the location of the interface depends on the mesh, such that it is always one cell away from the tip of the fan. Of the three dynamic meshing mechanisms in FLUENT, only smoothing and remeshing were used. Smoothing considers the movement of the fluid zone to be a spring-like deformation. Remeshing is used as a control tool that locally remeshes the cells if their new volume is smaller than the prescribed value of $5 \times 10^{-9}$ and only if these cells have a high skewness. This volume is specified based on the volume of the cells residing near the tip of the fan.

Region ' $b$ ' in the domain shown in Figure 13 is characterized by stationary fluid cells that interact with region 'a' through the fluid interface. Areas of higher cell density are included immediately in front of the heat source for improved resolution of the heater surface boundary layers. Because of the presence of the fluid interface described above, discontinuities are observed in contour plots at the interfacial line; however, it is noted that these discontinuities reflect a post-processing issue rather than errors in the numerical calculations. The mass and heat flux entering and leaving the fluid interface were verified to match.

\section{Model Setup}

The assumptions in the two-dimensional computational model include laminar, incompressible flow with no buoyancy or radiation contributions. First-order upwind discretization is used both for momentum and energy, with the SIMPLE scheme used for pressure-velocity coupling. Although a second-order scheme would yield better accuracy, these schemes were not explored due to constraints on computational time. In order to capture the flow field correctly, a very small time step is required. For all the results presented here, a time step of $1.6 \times 10^{-4} \mathrm{~s}$ was used. Thus, 100 steps in time were needed for one cycle of fan vibration.

The heat transfer coefficient is calculated based on the average fluid temperature and the average heat source surface temperature. The total duration of the simulation is selected such that the heat transfer coefficient has reached a steady value during this period. This took 10,000 time steps, which corresponds to approximately 40 hours of computation time per case on a dual processor (each $2.8 \mathrm{GHz}$ ) computer with $4 \mathrm{~GB}$ of memory. Mesh-size and time-step-size sensitivity were explored in a limited manner, and a coarser mesh run with larger time steps led to very similar results as those reported. The coarser mesh had 8,000 nodes, and run with time step size of $0.004 \mathrm{~s}$ (40 time steps per cycle of fan vibration.) The results presented below are obtained using a finer mesh with 20,000

\footnotetext{
${ }^{3}$ FLUENT 6.1.22, Fluent Inc. Lebanon, New Hampshire.
} 
nodes, and smaller time step of $0.0016 \mathrm{~s}$ (100 time steps per cycle of fan vibration.) The cycle-averaged and surface-averaged temperatures obtained with the coarse and fine meshes differed by $0.3^{\circ} \mathrm{C}$ in Case 1 . With the coarse mesh, the cycle-averaged heat transfer coefficients predicted on the plate were approximately $2.5 \%$ lower than the values obtained using the finer mesh.

\section{NUMERICAL RESULTS AND DISCUSSION}

Case $1,(\mathrm{a}=25 \%, \mathrm{~d}=0.64 \mathrm{~cm})$

This is the case for which the greatest heat transfer would be expected, since it considers the smallest fan to heat source separation and the largest fan amplitude. Four regions of circulation can be seen in Figure 14. The two smaller cells near the heat source are characterized by stronger velocities due to their proximity to the tip of the moving beam. These smaller, symmetric circulation zones above and below the vibrating beam are observed as the beam moves up and down, forcing fluid to displace more rapidly as the beam passes a given position on the heat source. The piezoelectric fan in Figure 14, shown at the undeflected position at this instant, has just returned from the upper extreme position and is traveling downwards. The closeness of the fan to the heat source leads to relatively high velocities and heat transfer coefficients (averaged over one full deflection cycle) relative to other cases with larger fan to heat source distances (to be discussed below). The larger circulation zones in Figure 14 entrain a larger amount of fluid and are also in contact with the outer boundaries of the domain which are at room temperature. The smaller circulation zones, on the other hand, are in direct contact with the heat source and are at a higher temperature than the fluid in the larger circulation zones, as shown in Figure 15.

\section{Case 2, $(\mathrm{a}=10 \%, \mathrm{~d}=2.54 \mathrm{~cm})$}

The second case considered, characterized by a large fan to heat source distance of $2.54 \mathrm{~cm}$ and a small amplitude ratio of $10 \%$, is expected to have the lowest heat transfer rates of the three cases. As the beam deflects in the upper and lower directions, vortices form and are shed away from the beam tip towards the stationary heat source (Figure 16). These vortices alternate with the direction of beam deflection, as occurs during vortex shedding in the flow around a cylinder. Pressure gradients induced by the displaced beam push the vortices toward the heat source causing fluid near the plate to be forced upward or downward, in an alternating fashion, into the larger circulation present in the fluid domain. The fluid loses much of its momentum as it reaches the heat source resulting 
in decreased heat transfer rates. The frequency of vortex shedding is twice the frequency of the fan, i.e., two vortices are shed during one full cycle of fan vibration. The two standing circulation regions of fluid near the heat source that were noticed in Case 1 are not realized in Case 2. On the other hand, the two larger circulation zones farther away from the fan are similar to those in Case 1.

Creation of vortices due to beam deflection was studied by Ihara and Watanabe (1994), who visualized the development of vortices and observed their movement. Figure 17 shows the computed vector field compared with the visualization from Ihara and Watanabe. The dashed line in the photograph in Figure 17, which is the third horizontal line from top, shows the location of the vibrating beam used in the experiment. The horizontal white lines were used to aid in measurement of the beam deflection; the distance between consecutive lines was $25 \mathrm{~mm}$. The visualization was carried out using a smoke wire, placed $3 \mathrm{~mm}$ downstream of the beam. Instead of piezoelectric fans, however, a simple cantilevered polyvinylchloride beam was excited at its resonance frequency of 21.7 Hz. The flexible portion of the beam was $90 \mathrm{~mm}$ in length, and had an amplitude of $35 \mathrm{~mm}$ at the tip. Although the present configuration differs from the experiment in Ihara and Watanabe (1994) in the presence of the heat source in the model as well as in the type and size of the oscillating beam, the vortex development and movement in Configuration B is qualitatively similar to the visualizations presented in Ihara and Watanabe (1994). As illustrated in Figure $18^{4}$, when the fan-to-heat source distance is decreased, however, these vortices are no longer shed from the fan tip, but instead contribute to the local fan tip circulation regions observed in Case 1 . It is noted that the flow field for Case 2 with the larger fan-to-heat source separation is markedly different from that for Case 1. As a result of the large circulation regions present, the heated fluid mixes with the bulk fluid domain more easily and does not recirculate in localized heated fluid regions. In contrast, the fluid was locally stirred in front of the heat source in Case 1 rather than being removed from the region. However, the heat transfer coefficient is lower for Case 2 due to the much smaller air velocities present in the vicinity of the heat source in this case.

\footnotetext{
${ }^{4}$ Photograph reproduced with publisher's permission from Ihara A., and Watanabe H., 1994, “On the Flow Around Flexible Plates, Oscillating With Large Amplitude,” Journal of Fluids and Structures, Vol 8, pp. 601-619.
} 
Case $3(\mathrm{a}=\mathbf{1 7 . 5 \%}, \mathrm{d}=\mathbf{1 . 5 9} \mathrm{cm})$

This case considers intermediate values of the fan to heat source separation and fan amplitude (Table 2), and appears to result in a flow field more like that of Case 1 (results not shown). While no discrete transition from standing circulation regions to vortex shedding was identified from the three cases considered in this study, it is likely that there is a transitional flow regime for conditions that lie between those of Cases 1 and 2 .

\section{COMPARISON OF PREDICTIONS AND EXPERIMENTS}

A comparison of the three cases studied in the computations reveals a decrease in heat transfer coefficient with increasing distance between the fan and heat source and decreasing amplitude ratio, as expected. Predicted convective heat transfer coefficients from this study are compared to the experimental results in Figure 19.

Since the numerically predicted heat transfer coefficients do not account for the contribution due to radiation, the radiative contribution determined in the experiments was subtracted from the experimentally determined effective heat transfer coefficients for this comparison. While satisfactory agreement is obtained for Cases 1 and 3, the simplified two-dimensional numerical model did not satisfactorily simulate Case 2. The main cause of this discrepancy is the two-dimensionality of the numerical model, which does not capture the three-dimensional flow field present in the experiments, which is expected to be especially pronounced in Case 2. The comparisons do show that a two-dimensional simplification can be satisfactory for small gaps. The flow pattern observed for Case 1 (small gap) and Case 3 (intermediate gap) are similar, whereas for Case 2 (large gap), a very different flow field is realized. When the gap is small, the flow induced by the piezofan is more localized and relatively two-dimensional in the vicinity of the heat source. The height of the heat source is three times the width of the piezofan; thus, decreasing the gap between the fan and the heat source makes the flow more two-dimensional in the vicinity of the heat source. Ideally, the experiments in this comparison should employ a very wide fan. The height of the heat source should also match the width of the piezofan. This would render the flow induced by the fan to be truly twodimensional. However, this is not experimentally feasible. On the other hand, a three-dimensional numerical model could be used, but such a model would be prohibitively expensive computationally (estimated to be months of run time for a single case). Alternative approaches to 3D modeling are being explored by the authors in ongoing work.

Also, the heat transfer coefficients in both the predictions and experiments were based on the temperature difference between the heat source surface (average) and the ambient in the enclosure. The ambient temperature in 
the experiments was measured with a single thermocouple suspended inside the enclosure, while in the simulation, the average fluid temperature inside the entire domain was used. Uncertainties in the temperatures, especially that of the enclosure ambient, can lead to differences between the experiment and prediction.

\section{CONCLUSIONS}

Piezoelectric fans have been shown to be a viable solution for the thermal management of low-power electronics in applications such as laptops, cell phones, PDAs and LED packages. The fans reduced the temperature of the heat source by as much as $37.4^{\circ} \mathrm{C}$, from values in natural convection of $70.6^{\circ} \mathrm{C}$. Among the parameters investigated, frequency offset, fan amplitude, and the distance between the fan and heat source have the greatest impact on the cooling capability of these fans; of these, frequency offset is the most significant. Even a small frequency offset of $5 \%$ decreased cooling capacity of the fan by more than $10^{\circ} \mathrm{C}$. Piezoelectric fan operation is modeled using an oscillating wall boundary condition with large deflection, and the resulting flow and temperature fields studied. Effects of changes in the fan to heat source distance and the fan deflection amplitude are investigated and compared against experimental results. It is observed that local circulation regions develop when the heat source is close to the fan tip. However, a different flow regime involving vortex shedding develops as this distance increases. The difference in these flow regimes result in significant differences in the heat transfer rates obtained. Transfer functions are presented for three different practical orientations of the fan and heat source. These transfer functions can be used in combination in the implementation of piezoelectric fans in cooling applications.

\section{Acknowledgments}

Financial support for this work from GELcore is gratefully acknowledged. 
Table 1. LSL and USL values for the parameters investigated in Configurations A, B, and C.

\begin{tabular}{ccc|cc|cc}
\hline & \multicolumn{2}{c|}{ Configuration A } & \multicolumn{2}{c}{ Configuration B } & \multicolumn{2}{c}{ Configuration C } \\
& LSL & USL & LSL & USL & LSL & USL \\
\cline { 2 - 7 } Fan length [cm] & 6.86 & 7.62 & 6.86 & 7.62 & 6.86 & 7.62 \\
Amplitude ratio at resonance [\%L] & 10 & 25 & 10 & 25 & 10 & 25 \\
Frequency offset [\% \%r] & -5 & 5 & -5 & 5 & -5 & 5 \\
Fan offset [cm] & 0 & 1.27 & -1.27 & 1.27 & N/A & N/A \\
Distance to fan tip [cm] & 0.64 & 2.54 & 0.64 & 1.9 & 0.64 & 1.9 \\
\hline
\end{tabular}


Table 2. Parameter values for the cases considered in the numerical analysis.

\begin{tabular}{lccc}
\hline & \multicolumn{3}{c}{ Case } \\
\cline { 2 - 4 } & 1 & 2 & 3 \\
\cline { 2 - 4 } Fan tip to heat source distance, $\mathrm{d}[\mathrm{cm}]$ & 0.64 & 2.54 & 1.59 \\
Amplitude ratio at resonance, $\mathrm{a}[\% \mathrm{~L}]$ & 25 & 10 & 17.5 \\
\hline
\end{tabular}


Table 3. Piezoelectric fan properties.

\begin{tabular}{cc}
\hline Piezoelectric patch material & PZT \\
Piezoelectric patch thickness & $0.05 \mathrm{~cm}$ \\
Piezoelectric patch length & $2.9 \mathrm{~cm}$ \\
Shim material & Mylar \\
Shim thickness & $0.025 \mathrm{~cm}$ \\
Total piezoelectric fan length (L) & $7.62 \mathrm{~cm}$ \\
Width of the piezoelectric fan & $1.3 \mathrm{~cm}$ \\
Resonance frequency & $62 \mathrm{~Hz}$ \\
\hline
\end{tabular}




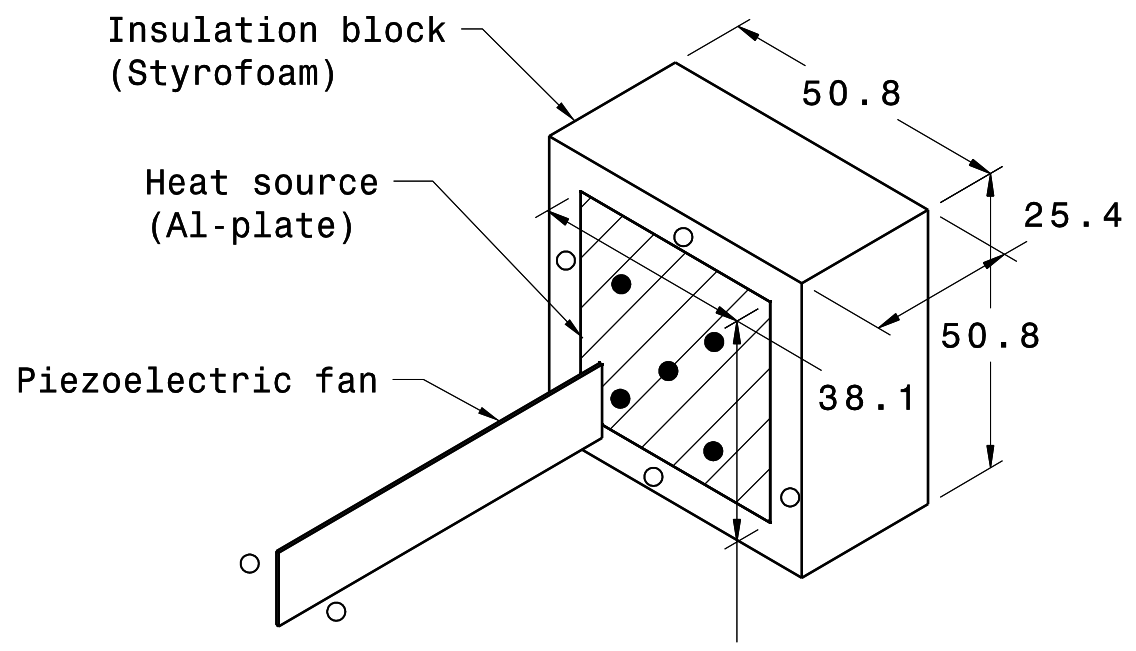

38.1

$$
\text { Thermocouples } \bullet \text { attached } \circ \text { free }
$$

Figure 1. Schematic diagram of the heat source and thermocouple locations. All dimensions are in $\mathrm{mm}$. 


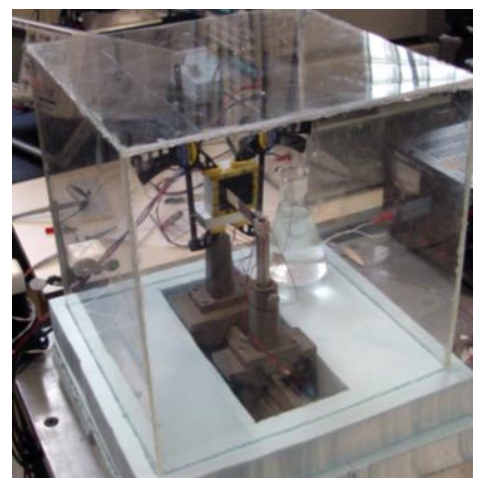

(a)

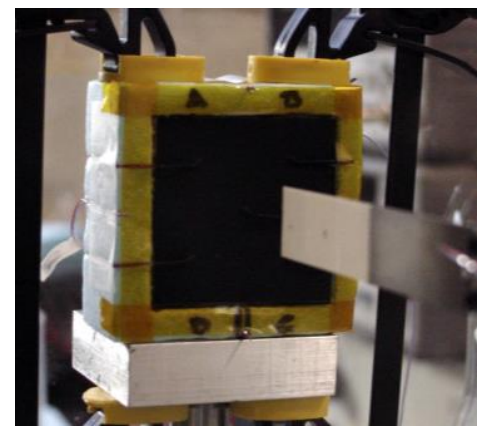

(b)

Figure 2. Photographs of a) the experimental setup, and b) detail of the heat source and fan arrangement. 

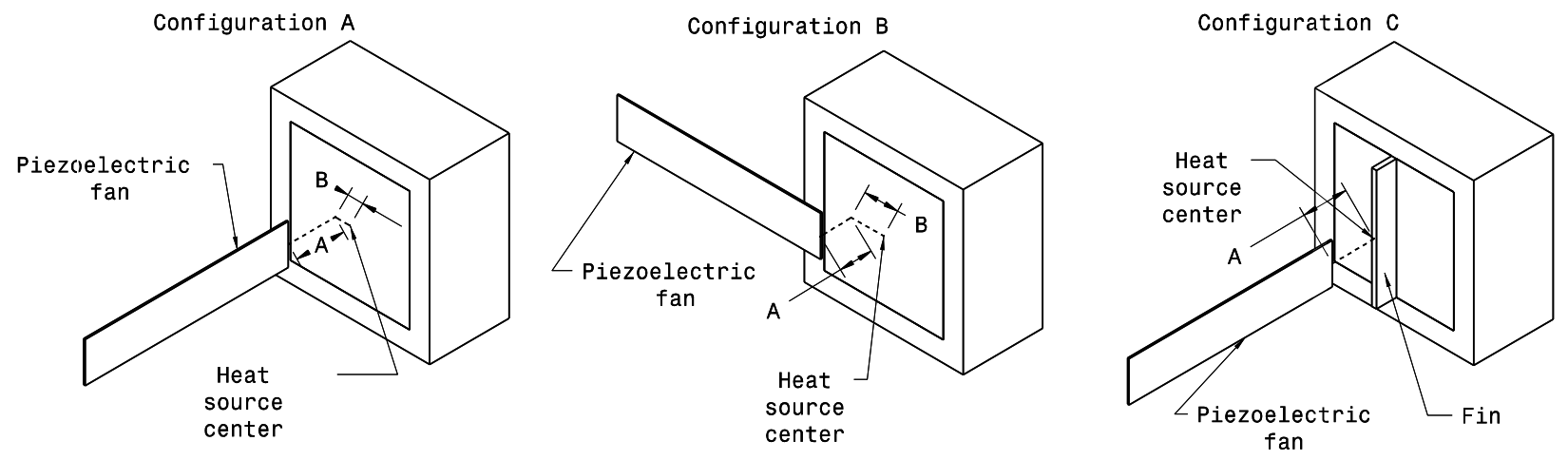

Figure 3. Schematic of different experimental configurations: Configuration A) Fan in front, Configuration B) Fan to the side, and Configuration $C$ ) Fan in front with fin. Definitions of two of the parameters studied for different configurations, distance to fan tip (A), and fan offset (B), are also denoted. 


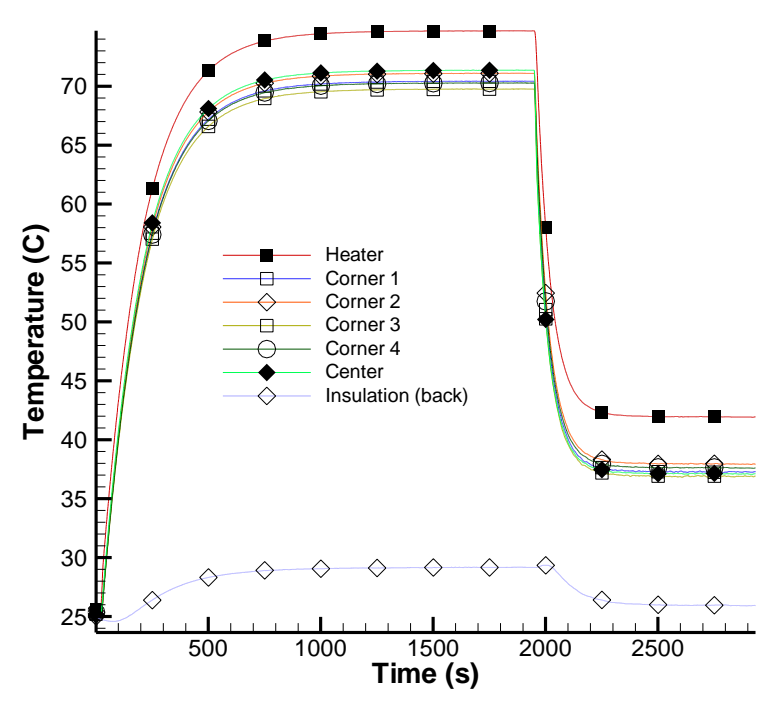

(a)

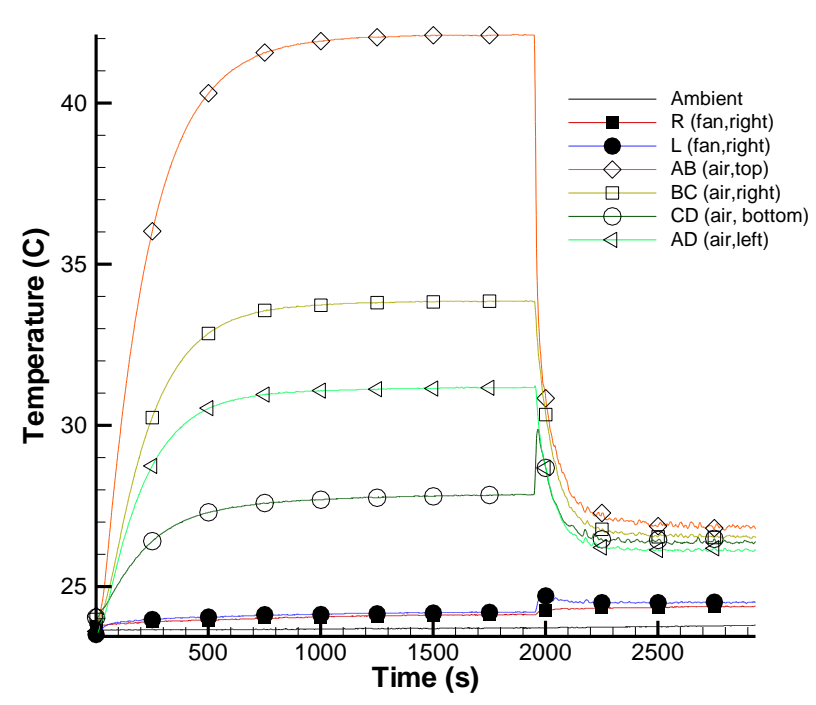

(b)

Figure 4. Temperatures from a sample test in Configuration A: (a) temperatures on the heat source (four corners, center of heat source, between heater and insulation, and back of insulation), and (b) temperatures in air ( $R$ and $L$ are on both sides of the fan, while the other four are in air close to the heat source, Figure 1). 


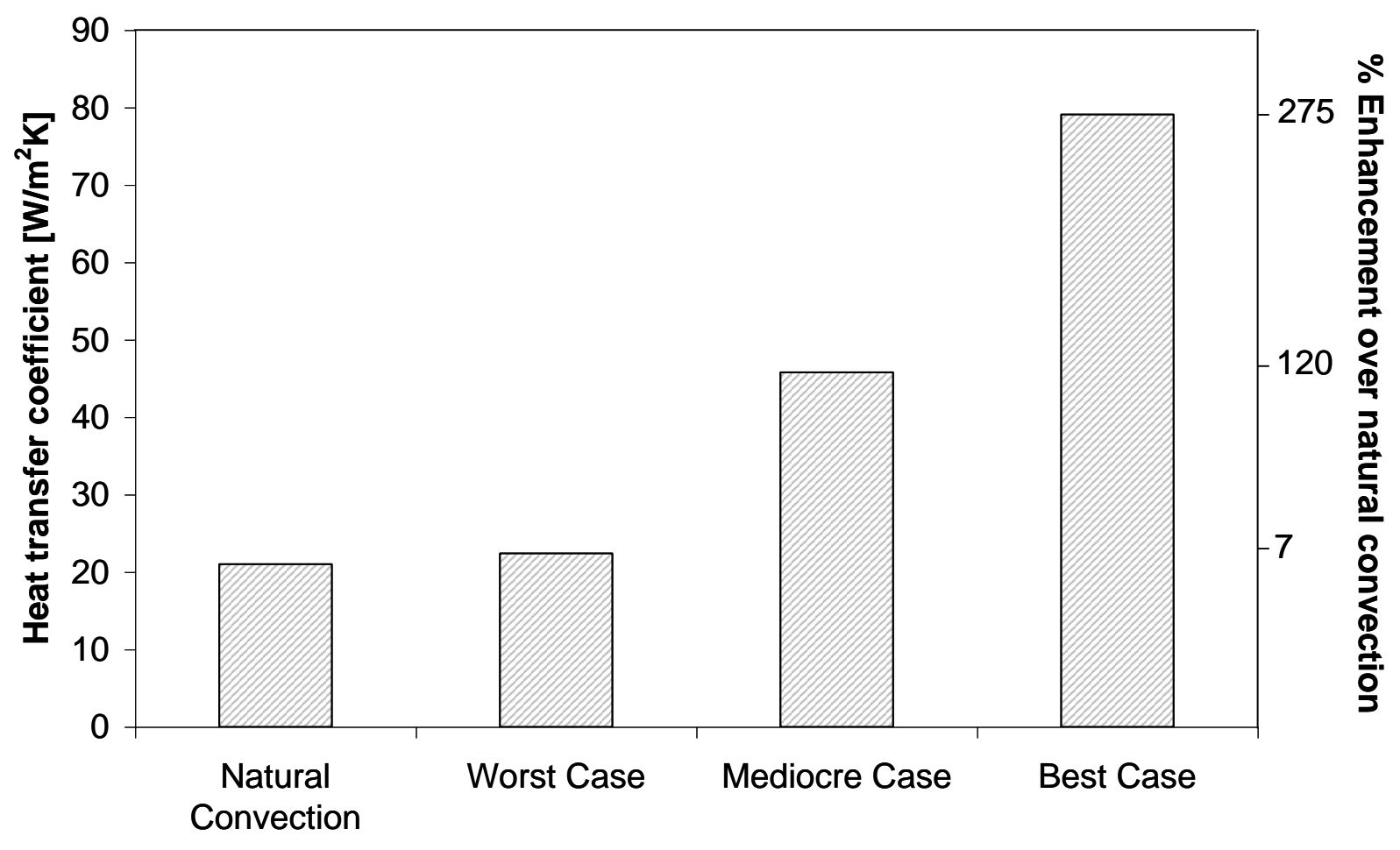

Figure 5. Heat transfer coefficients from three different experiments in Configuration A, along with the natural convection value. 


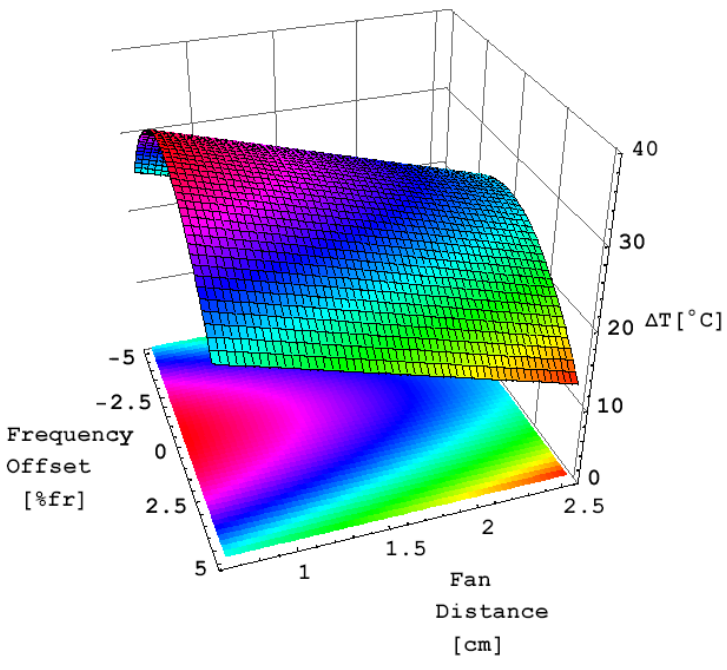

(a)

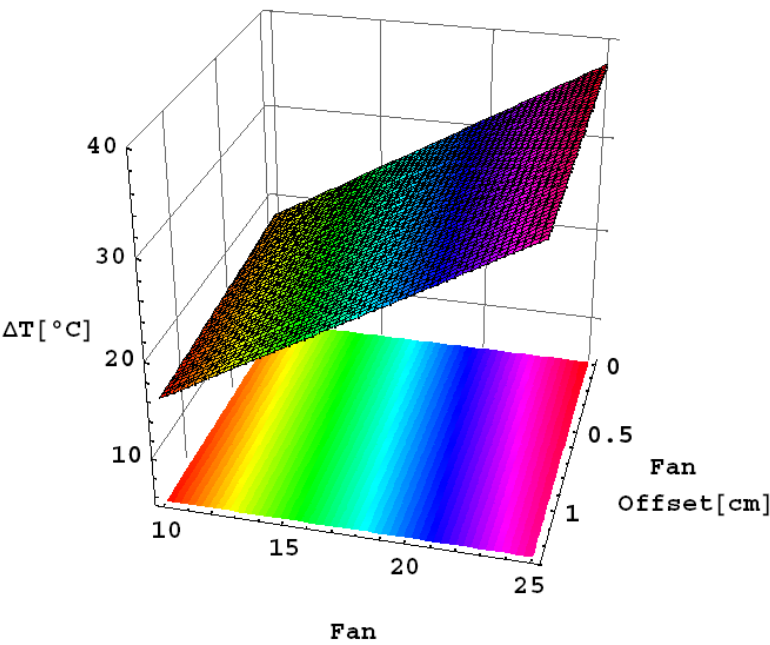

Amplitude

$[\% \mathrm{~L}]$

Figure 6. Heat source temperature differential for Configuration A: (a) for varying frequency offset and fan-heat source distance (for a $7.62 \mathrm{~cm}$ fan with an amplitude of $25 \%$ and no fan offset), and (b) for varying fan offset and fan amplitude (for a $7.62 \mathrm{~cm}$ fan with a fan distance of $0.64 \mathrm{~cm}$ and no frequency offset). In this and all similar shadow 3D plots in this paper, a projection of the surface on a horizontal plane is also included as a visual aid. 


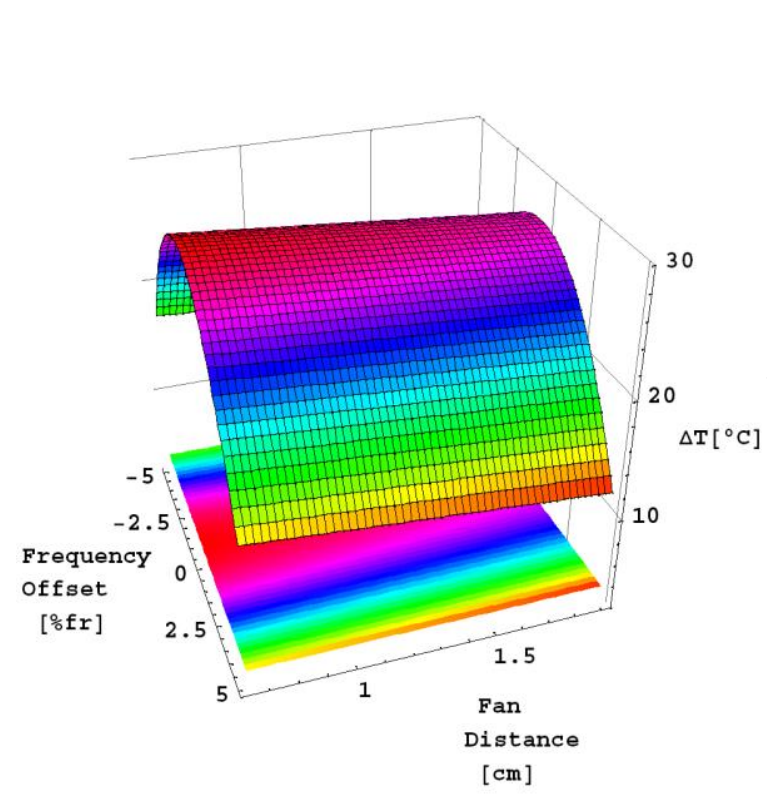

(a)

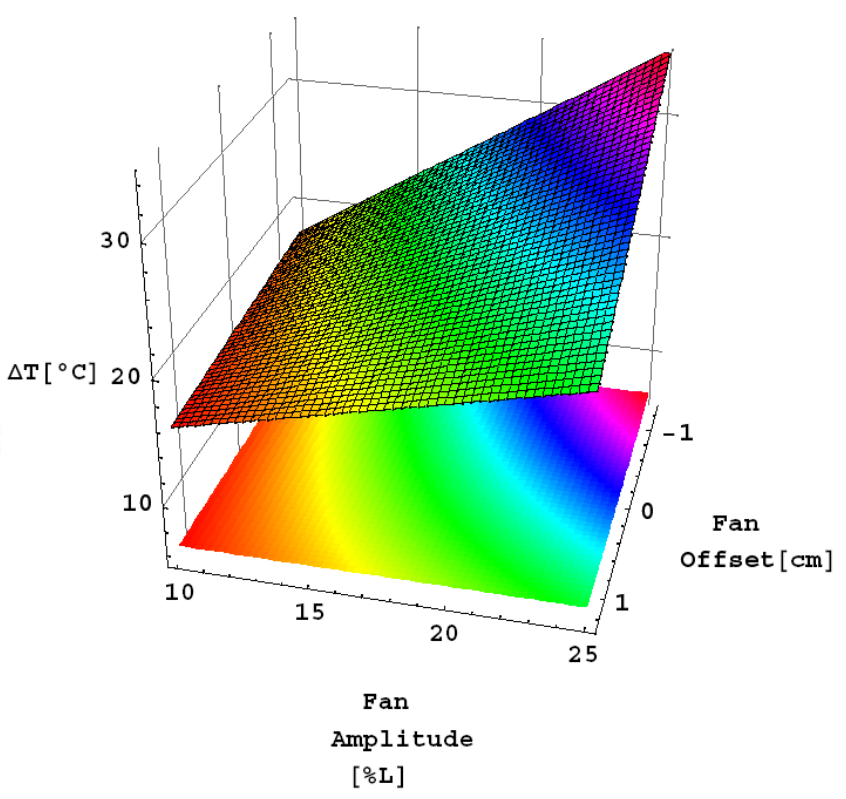

(b)

Figure 7. Heat source temperature differential for Configuration B: (a) for varying frequency and fan-heat source distance (for a $7.62 \mathrm{~cm}$ fan with an amplitude of $25 \%$ and no fan offset), and (b) for varying fan amplitude and fan offset (for a 7.62 $\mathrm{cm}$ fan with a fan distance of $0.64 \mathrm{~cm}$ and no frequency offset). 


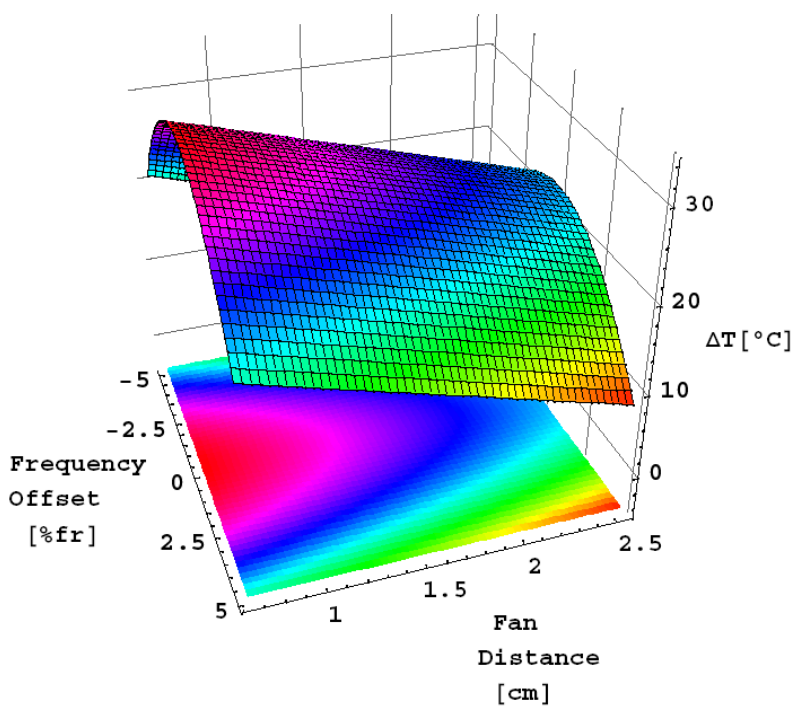

(a)

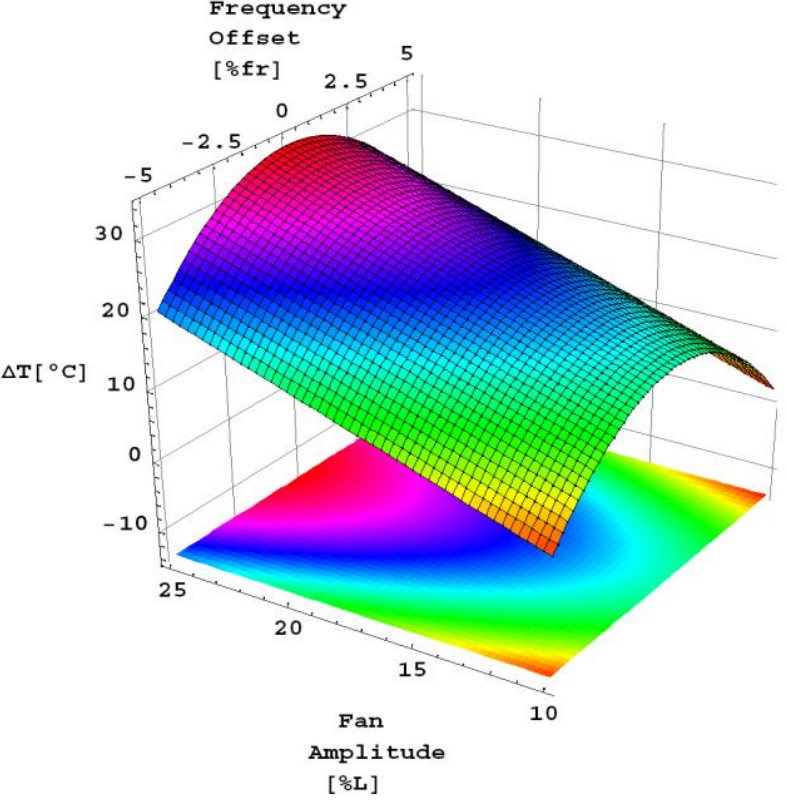

(b)

Figure 8. Heat source temperature differential for Configuration C: (a) for varying frequency and fan-heat source distance (for a $7.62 \mathrm{~cm}$ fan with an amplitude of $25 \%$ ), and (b) for varying frequency and fan amplitude (for a $7.62 \mathrm{~cm}$ fan with a fan distance of $0.64 \mathrm{~cm}$ ). 


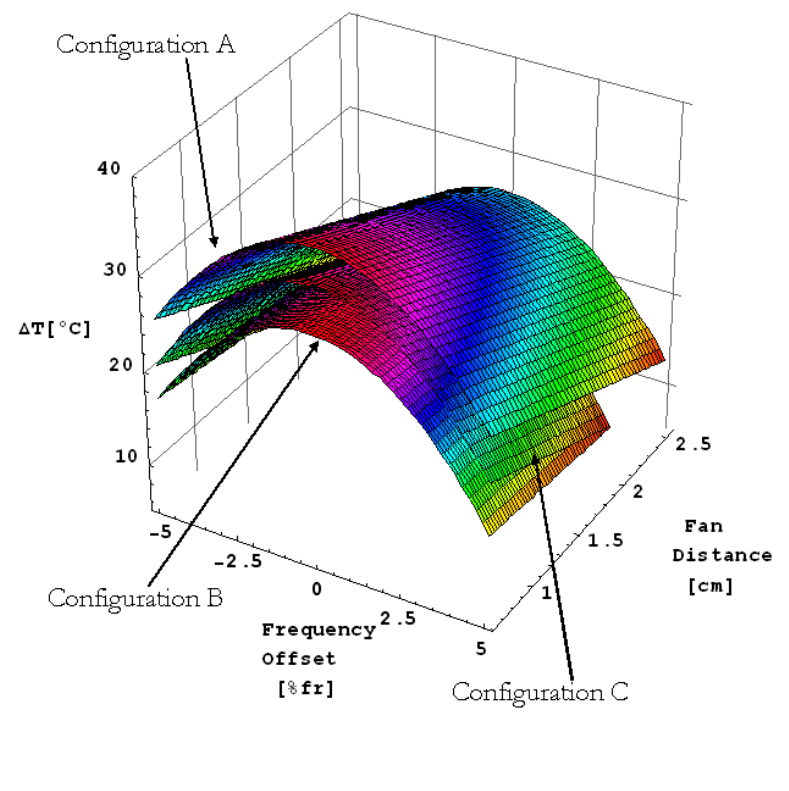

Figure 9. Comparison of DOE results from Figures $6 a, 7 a$, and $8 a$. 


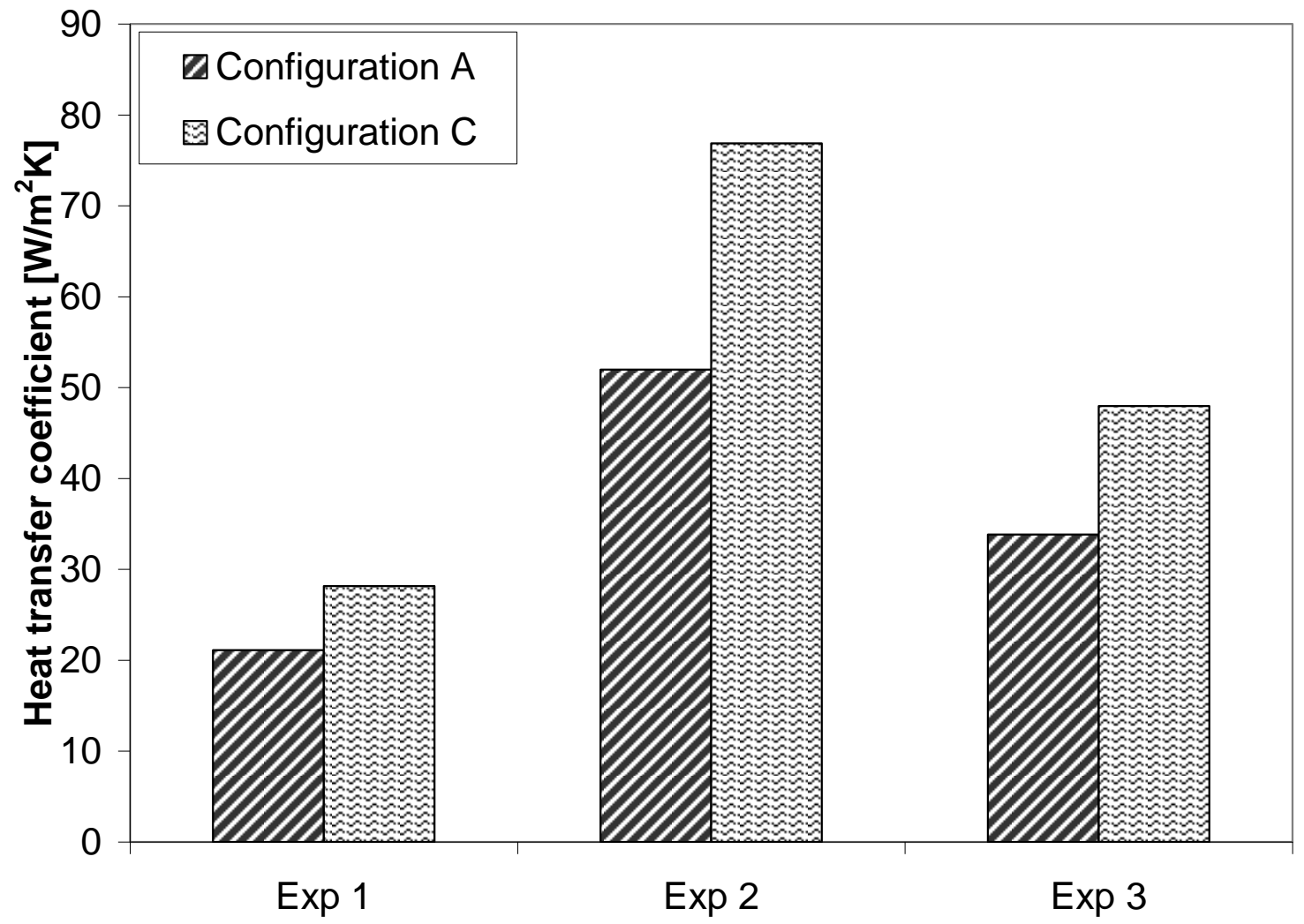

Figure 10. Comparison of three individual experiments from Configurations $A$ and $C$ with matching parameters, illustrating the effect of inclusion of the fin. 


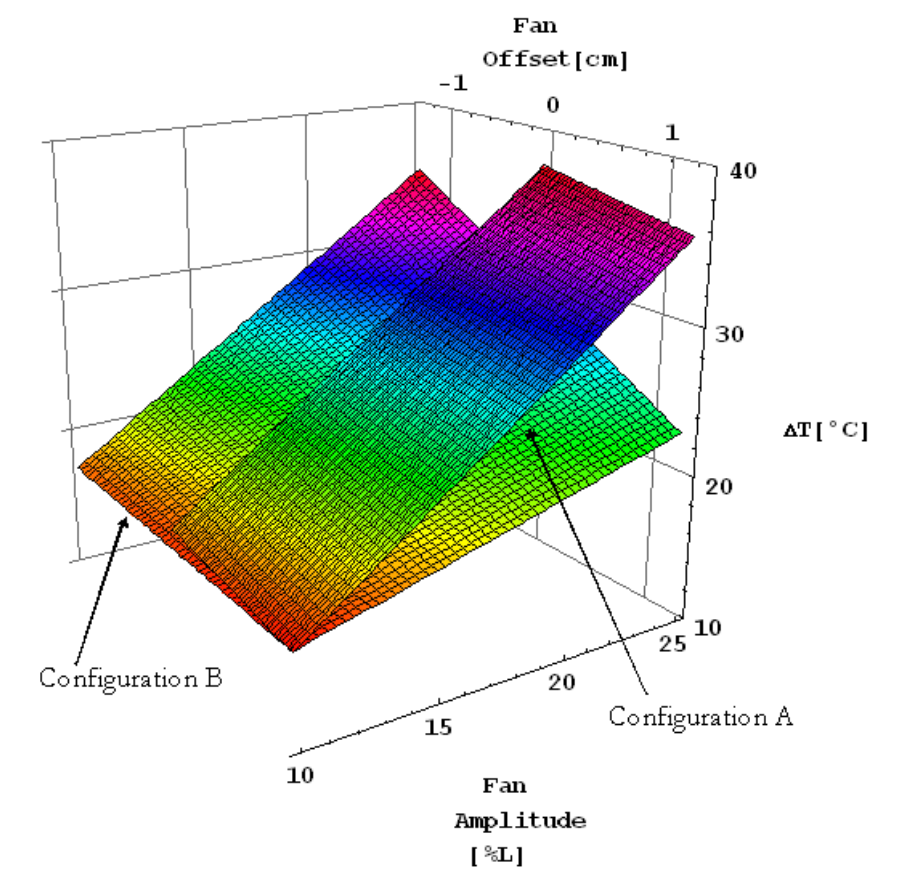

Figure 11. Comparison of DOE results from Figures $6 b$ and $7 b$. 

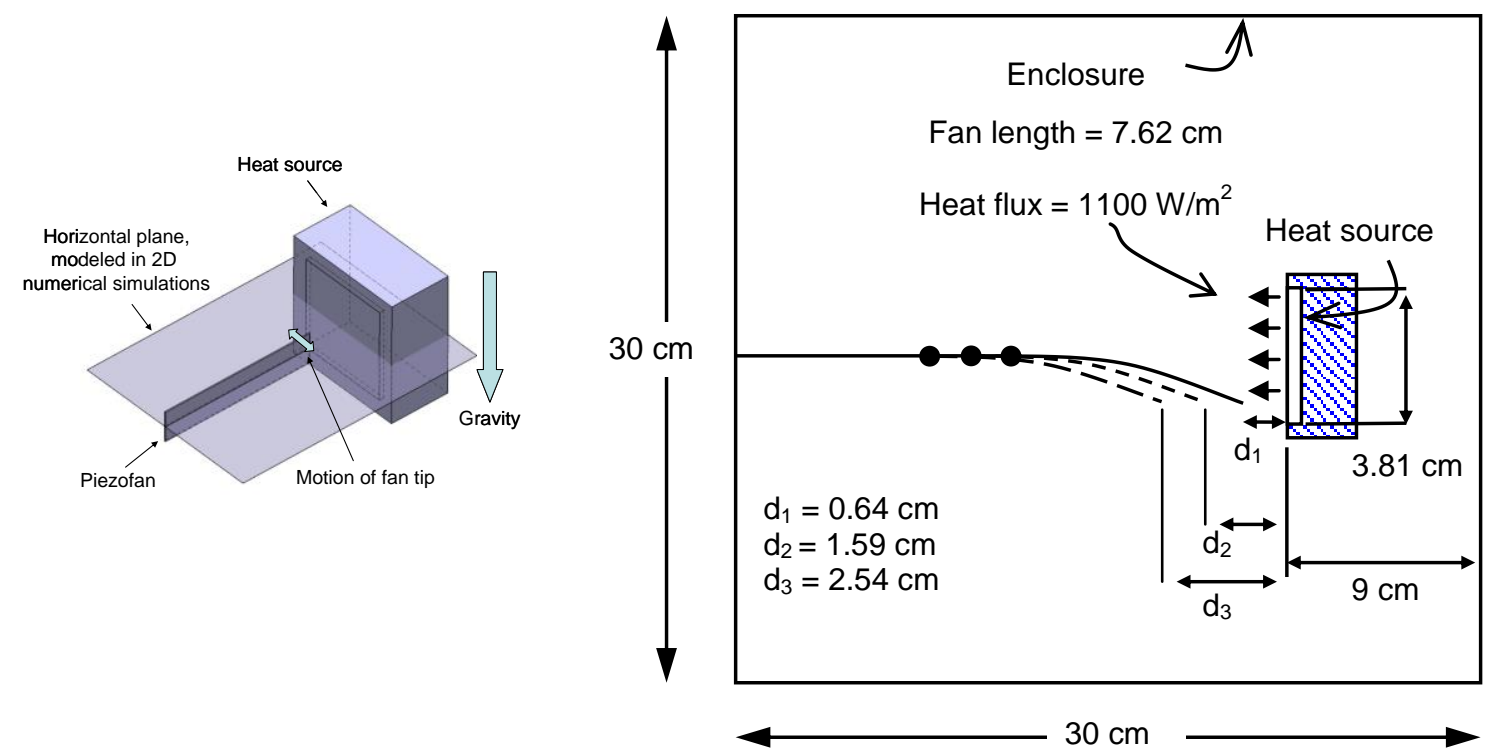

Figure 12. Conditions and geometry of the three cases studied; all three fan locations, at distances $d_{1}, d_{2}$ and $d_{3}$ from the heat source are shown. The two-dimensional numerical model considers the horizontal mid-plane of the experiments; the direction of gravity is normal to this plane as shown. 


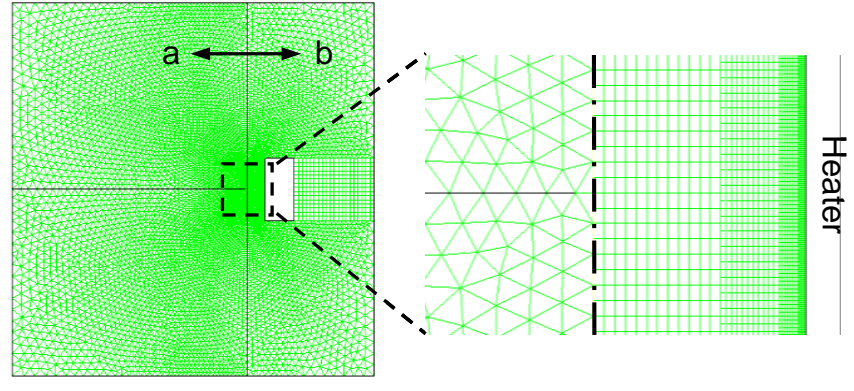

Figure 13. Mesh generation boundaries and regions. 

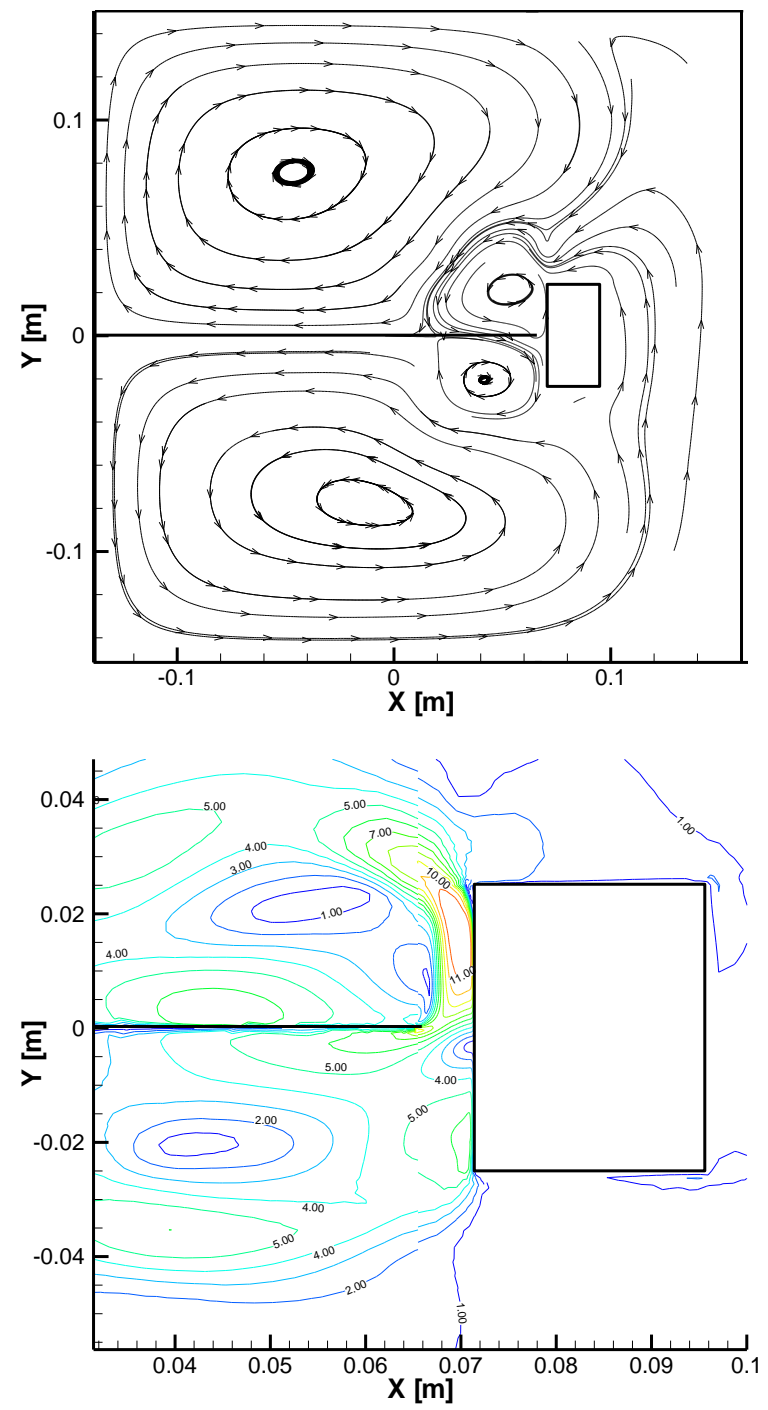

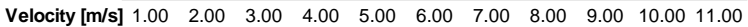

Figure 14. Streamlines and contours of velocity magnitude for Case $1(a=25 \%, d=0.64 \mathrm{~cm})$ at time $=1.68 \mathrm{~s}$. At this instant the fan is passing its original position and traveling downward. 


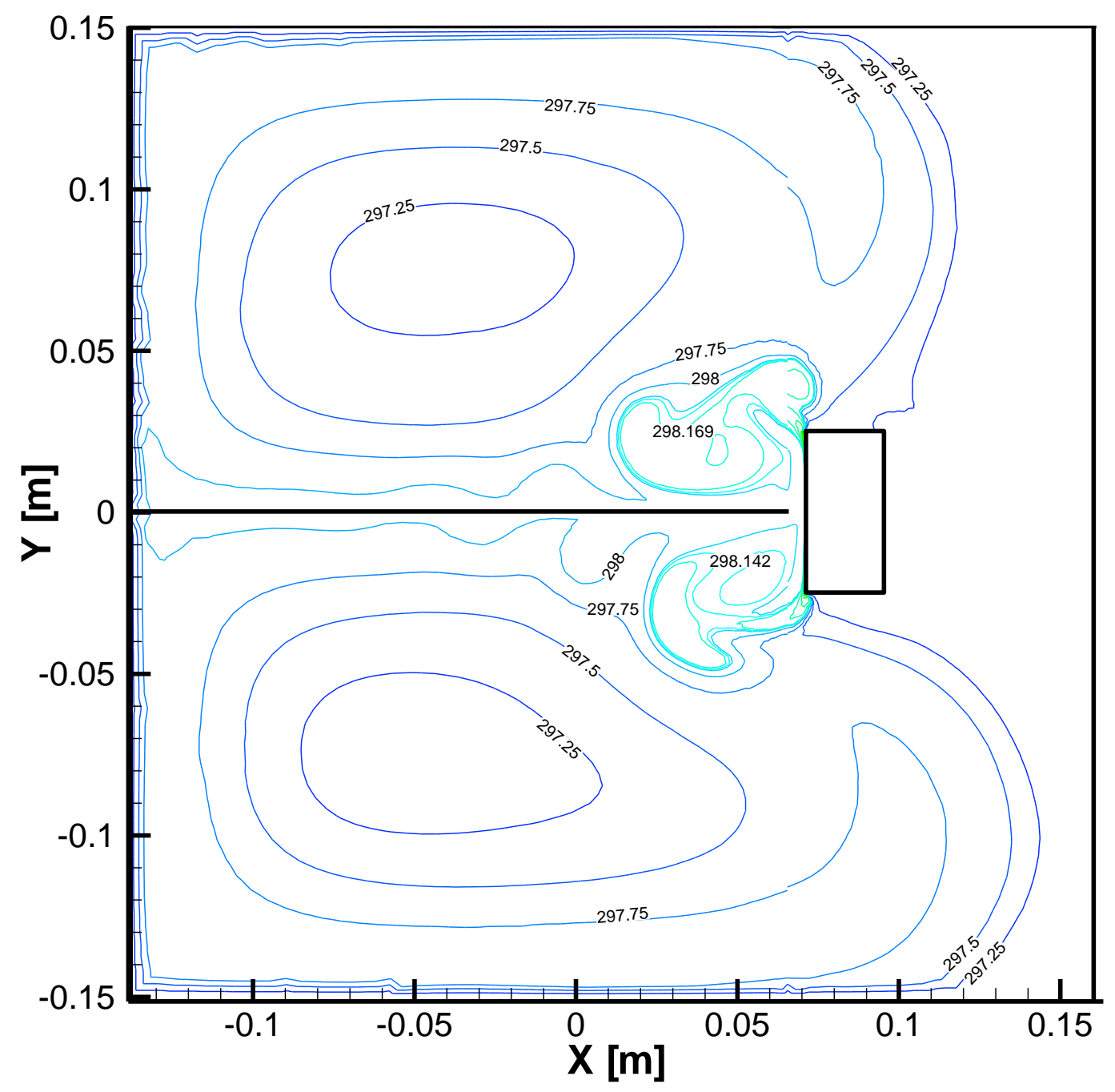

Figure 15. Temperature contours for Case $1(a=25 \%, d=0.64 \mathrm{~cm})$ at time $=1.68 \mathrm{~s}$. At this instant the fan is passing its original position and traveling downward. 


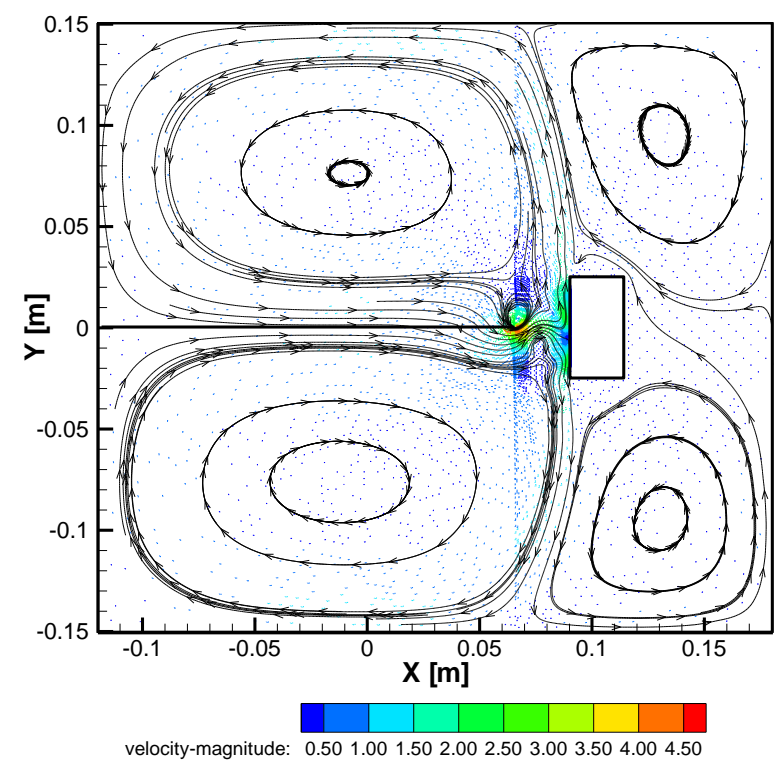

Figure 16. Velocity vectors and streamlines for Case $2(a=10 \%, d=2.54 \mathrm{~cm})$ at time $=3.2 \mathrm{~s}$. At this instant the fan is passing its original position and traveling downward. 


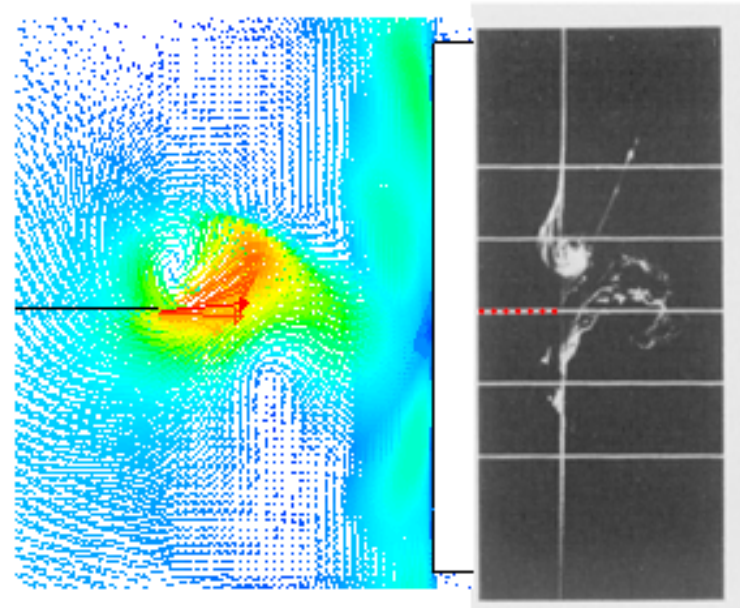

Figure 17. Comparison of the computed velocity field with the experimental flow visualization of Ihara and Watanabe (1994); photograph reproduced with publisher's permission. 


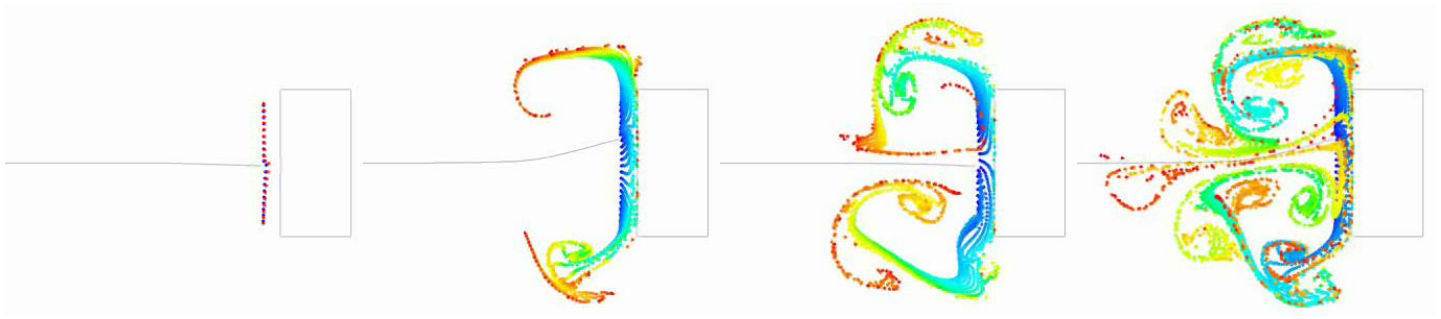

(a)

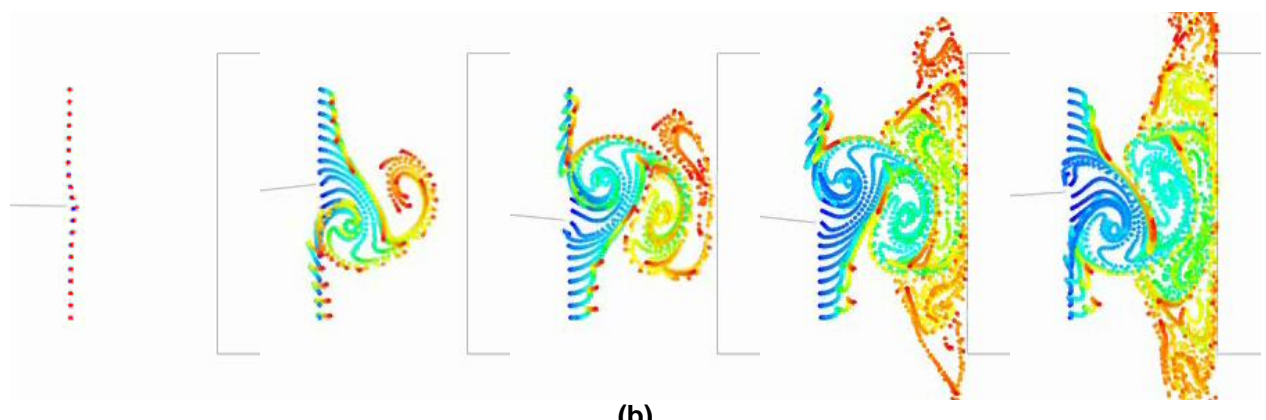

(b)

Figure 18. Numerical flow visualizations for (a) Case $1(a=25 \%, d=0.64 \mathrm{~cm})$, and (b) Case $2(a=10 \%, d=2.54 \mathrm{~cm})$. 


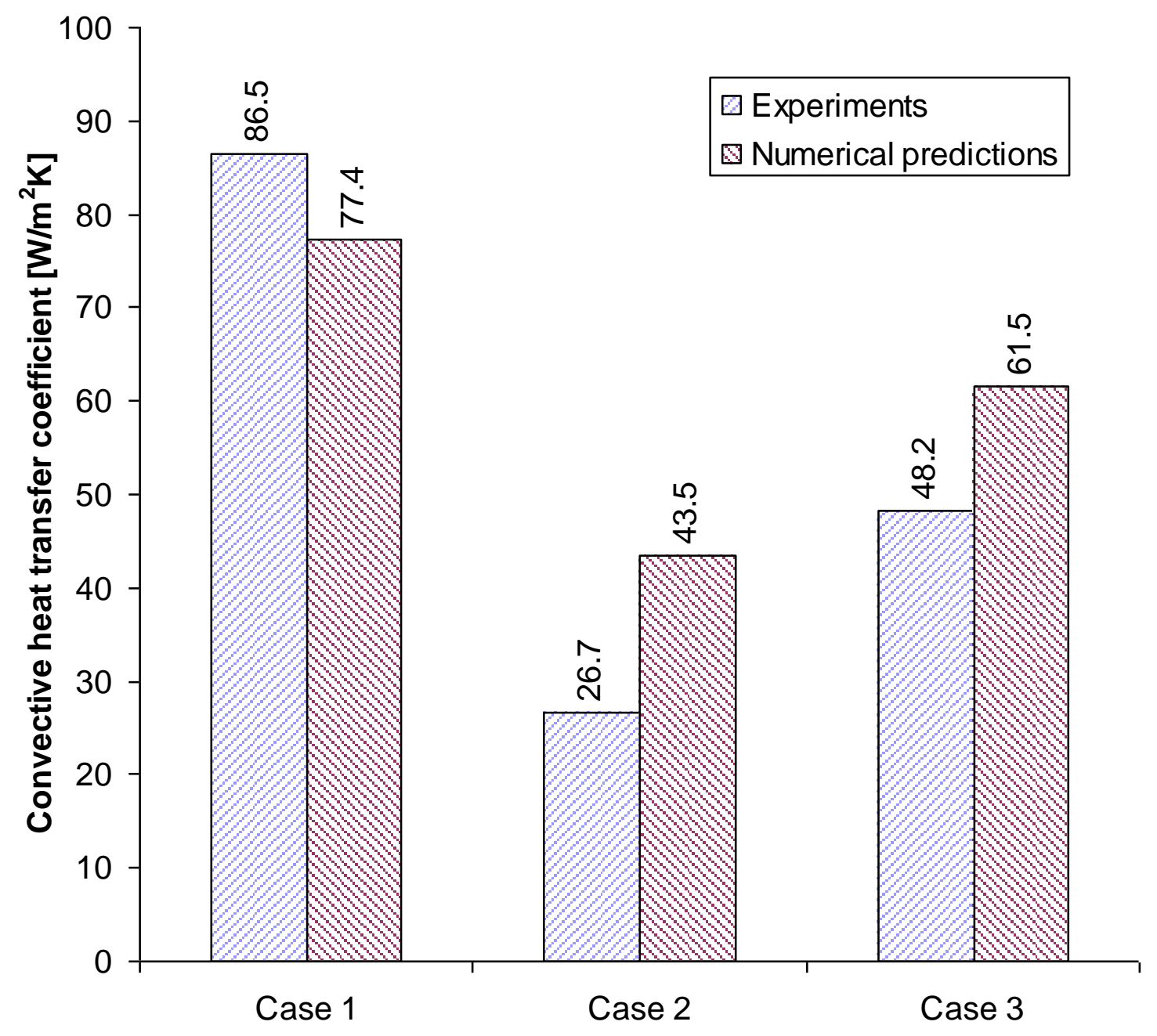

Figure 19. Comparison of predicted and experimentally determined heat transfer coefficients. 


\section{REFERENCES}

Açıkalın T., Raman A., and Garimella S.V., "Two-Dimensional Streaming Flows Induced by Resonating, Thin Beams," J. Acoust. Soc. Am., Vol. 114, pp. 1785-1795, 2003.

Açıkalın T., Wait S. M., Garimella S. V. and Raman A., "Experimental Investigation of the Thermal Performance of Piezoelectric Fans," Heat Transfer Engineering, Vol. 25, pp. 4-14, 2004.

Basak S., Raman A., and Garimella S. V., "Dynamic Response Optimization of Piezoelectrically Excited Thin Resonant Beams," ASME Journal of Vibration and Acoustics, Vol. 127, pp. 18-27, 2005.

Buermann P., Raman A., and Garimella S. V., "Dynamics and Topology Optimization of Piezoelectric Fans," IEEE Transactions on Components and Packaging Technologies, Vol. 25, pp. 113-121, 2002.

Ihara A., and Watanabe H., "On the Flow around Flexible Plates, Oscillating With Large Amplitude,” J. Fluid. Struct., vol. 8, pp. 601-619, 1994.

Loh B. G., Hyun S., Ro P. I. and Kleinstreuer C., "Acoustic Streaming Induced by Ultrasonic Flexural Vibrations and Associated Enhancement of Convective Heat Transfer,” J. Acoust. Soc. Am., vol. 111, 875-883, 2002.

Schmidt R. R., "Local and Average Transfer Coefficients on a Vertical Surface Due to Convection from a Piezoelectric Fan," Procs. ITHERM, pp. 41- 49, 1994.

Schmidt S. R., and Launsby R. G., Understanding Industrial Designed Experiments, $4^{\text {th }}$ edition, Colorado Springs, CO, 2003

Toda M, "Voltage-induced Large Amplitude Bending Device-PVF 2 Bimorph-Its Properties and Applications," Ferroelectrics, vol. 32, pp. 127-133, 1981.

Toda M.,, "Theory of Air Flow Generation by a Resonant Type $\mathrm{PVF}_{2}$ Bimorph Cantilever Vibrator," Ferroelectrics, vol. 22, pp. 911-918, 1979.

Wait S. M., Açıkalın T., Garimella S. V., and Raman A., "Piezoelectric Fans for the Thermal Management of Electronics," Procs. Sixth ISHMT/ASME Heat and Mass Transfer Conference, Kalpakkam, India, Paper No. HMT-2004-C76, pp. 447-452, January $5-7,2004$.

Yoo J. H., Hong J. I., and Cao W., "Piezoelectric Ceramic Bimorph Coupled to Thin Metal Plate as Cooling Fan for Electronic Devices," Sensor Actuat. A Phys, vol. 79, pp. 8-12, 2000. 